\title{
Achieving Efficiency in Dynamic Contribution Games
}

\author{
By JAKŠA CVITANIĆ AND GeORge GeOrgiadis*
}

\begin{abstract}
We analyze a game in which a group of agents exerts costly effort over time to make progress on a project. The project is completed once the cumulative effort reaches a prespecified threshold, at which point it generates a lump-sum payoff. We characterize a budget-balanced mechanism that induces each agent to exert the first-best effort level as the outcome of a Markov perfect equilibrium, thus eliminating the free-rider problem. We also show how our mechanism can be adapted to other dynamic games with externalities, such as strategic experimentation and the dynamic extraction of a common resource. (JEL C73, D62, D82, Q31)
\end{abstract}

T eam problems are ubiquitous in modern economies as individuals and firms often need to collaborate in production and service provision (Ichniowski and Shaw 2003). Moreover, the collaboration is often geared towards achieving a particular set of objectives, i.e., towards completing a project (Harvard Business Review 2004). For example, in early-stage entrepreneurial ventures (e.g., startups), individuals collaborate over time to create value, and the firm generates a payoff predominantly when it goes public or is acquired by another corporation. Joint research and development and new product development projects also share many of these characteristics: corporations work together to achieve a common goal, progress is gradual, and the payoff from the collaboration is harvested primarily after said goal is achieved, for example, once a patent is secured, or the product under development is released to the market. Unfortunately, as it is well known, such environments are susceptible to the free-rider problem (Olson 1965, Alchian and Demsetz 1972).

We study a game of dynamic contributions to a joint project (Admati and Perry 1991), which can be summarized as follows: At every moment each agent in the group chooses his (costly) effort level to progressively bring the project closer to completion. The agents receive a lump-sum payoff upon completion, and they discount time. An equilibrium feature of this game is that the agents exert greater effort

\footnotetext{
* Cvitanić: Division of the Humanities and Social Sciences, California Institute of Technology, 1200 E. California Blvd., Pasadena, CA 91125 (e-mail: cvitanic@hss.caltech.edu); Georgiadis: Kellogg School of Management, Northwestern University, 2001 Sheridan Rd., Evanston, IL 60208 (e-mail: g-georgiadis@kellogg.northwestern. edu). We are grateful to Attila Ambrus, Simon Board, Kim Border, Yeon-Koo Che, Larry Kotlikoff, Eddie Lazear, Fei Li, Albert Ma, Niko Matouschek, Moritz Meyer-ter-Vehn, Dilip Mookherjee, Andy Newman, Juan Ortner, Andy Skrzypacz, Chris Tang, Luke Taylor, and Glen Weyl, as well as to seminar audiences at Boston University, Northwestern University (Kellogg), Michigan State University, the University of Pennsylvania, INFORMS 2015, the 2015 Canadian Economic Theory Conference, the Annual 2015 IO Theory Conference, and the 2015 Midwest Economic Theory Conference for numerous comments and suggestions. We thank Maja Kos for excellent editorial assistance. J. Cvitanić acknowledges support from NSF grant DMS 10-08219.

Go to http://dx.doi.org/10.1257/mic.20160018 to visit the article page for additional materials and author disclosure statement $(\mathrm{s})$ or to comment in the online discussion forum.
} 
the closer the project is to completion. Therefore, by raising his effort, an agent can induce the others to raise their future efforts, which renders him better off; thus, efforts are strategic complements across time (Kessing 2007). By comparing the equilibrium strategies with the efficient ones, we identify two kinds of inefficiencies. First, because each agent is incentivized by his share of the project's payoff (rather than the entire payoff), in equilibrium he exerts inefficiently low effort. Second, because efforts are strategic complements, each agent front-loads his effort in order to induce others to raise their future efforts. The former has been extensively discussed in the literature, but the latter, while subtle, is also intuitive. For instance, startups often use vesting schemes to disincentivize entrepreneurs from front-loading effort, i.e., working hard early on and then walking away while retaining their stake in the firm.

We propose a budget-balanced mechanism that induces each agent to exert the efficient level of effort as the outcome of a Markov perfect equilibrium (MPE). The mechanism specifies for each agent flow payments that are due while the project is in progress and a reward that is disbursed upon completion. These payments are placed in a savings account that accumulates interest. The mechanism effectively rewards each agent with the entire payoff that the project generates, thus making him the full residual claimant, while the flow payments increase with progress so that the marginal benefit from front-loading effort is exactly offset by the marginal cost associated with larger flow payments in the future. The idea behind our mechanism can be viewed as a dynamic counterpart of the budget breaker in Holmström (1982), except that the savings account plays the role of the budget breaker in our setting.

Our mechanism resembles many of the features in the incentives structures commonly observed in startups. In particular, entrepreneurs usually receive a salary that is below the market rate (if any). The flow payments in our mechanism can be interpreted as the difference between what an individual earns in the startup and his outside option, i.e., what he would earn if he were to seek employment in the market. Finally, entrepreneurs typically own shares, so they are residual claimants. Alternatively, the flow payments can be interpreted as the cash investments that the entrepreneurs need to make in the project until they can raise capital. Based on the latter interpretation, our mechanism is resource feasible provided that the agents have sufficient cash reserves at the outset of the game to make the specified flow payments.

To distill the intuition, we start with the simplest possible framework, in which the project progresses deterministically, and the mechanism depends on the state of the project, but not on time. We later extend our model to incorporate uncertainty in the evolution of the project, and we characterize the efficient time-dependent mechanism. In this case, flow payments are specified as a function of both time and the state of the project, and terminal rewards as a function of the completion time. This mechanism specifies a terminal reward that decreases in the completion time of the project, and at every moment the flow payments are chosen so as to penalize the agents if the project is closer to completion than it should be on expectation if all agents were applying the first-best strategies. Intuitively, the former acts as a lever to mitigate the agents' incentives to shirk, while the latter as a lever to eliminate their incentives to front-load effort. An important difference relative to the 
deterministic mechanism is that with uncertainty, it is impossible to simultaneously achieve efficiency and ex post budget balance; instead, the efficient mechanism can only be budget balanced ex ante (i.e., in expectation). Finally, in the special case in which the project progresses deterministically, the efficient time-dependent mechanism specifies that the agents make zero flow payments on the equilibrium path (but positive off path), and as such, it is resource feasible even if the agents are credit constrained.

While we develop our mechanism in the context of dynamic contribution games, our mechanism can be readily adapted to other dynamic games with externalities. To illustrate the versatility of our framework, we consider two common dynamic games: first, a dynamic common resource extraction problem such as the one studied by Levhari and Mirman (1980) and Reinganum and Stokey (1985), and second, a strategic experimentation problem similar to that in Keller, Rady, and Cripps (2005) and Bonatti and Hörner (2011). In each case, we construct a mechanism that induces the agents to always choose the efficient actions as the outcome of an MPE. For the resource extraction problem, the mechanism specifies that each agent receives a flow subsidy that decreases as the resource becomes more scarce to neutralize his incentives to overharvest it. To achieve budget balance, each agent must pay a combination of an entry fee in exchange for access to the resource and a penalty fee that becomes due as soon as it is depleted. Similarly for the experimentation problem, the mechanism specifies that each agent pays a fee to enter the mechanism and receives a subsidy that is a function of the (public) belief about the risky project being lucrative.

Naturally, our paper is related to the literature on the static moral hazard in teams (Olson 1965; Holmström 1982; Ma, Moore, and Turnbull 1988; Bagnoli and Lipman 1989; Legros and Matthews 1993; and others). These papers focus on the free-rider problem that arises when each agent must share the output of his effort with other members of the team, but he alone bears its cost, and they explore ways to restore efficiency. Holmström (1982) shows that in a setting wherein a group of agents jointly produce output, there exists no budget-balanced sharing rule that induces the agents to choose efficient actions. Moreover, he shows how efficiency is attainable by introducing a third party who makes each agent the full residual claimant of output and breaks the budget using transfers that are independent of output. Legros and Matthews (1993) provide a necessary and sufficient condition for the existence of a sharing rule such that the team sustains efficiency. Their construction uses mixed strategies where all agents but one choose the efficient actions, and the last agent chooses the efficient action with probability close to 1 . However, a necessary condition for this mechanism to sustain efficiency is that the agents have unlimited liability.

More closely related to this paper is the literature on dynamic contribution games. Admati and Perry (1991) characterize an MPE with two players, and they show that efforts are inefficiently low relative to the efficient outcome. Marx and Matthews (2000) generalize this result to games with $n$ players, and they also characterize subgame perfect equilibria with non-Markov strategies. More recently, Yildirim (2006) and Kessing (2007) show that if the project generates a payoff only upon completion, then efforts become strategic complements across time. Our model is 
a generalized version of Georgiadis, Lippman, and Tang (2014) who consider the problem faced by a project manager who chooses the size of the project to be undertaken and has limited ability to commit to the project requirements at the outset of the game. Georgiadis (2015a) studies how the size of the group influences the agents' incentives and analyzes the problem faced by a principal who chooses the group size and the agents' incentive contracts to maximize her discounted profit. Last, Georgiadis (2015b) examines how commitment to a deadline and altering the monitoring structure in this class of games can mitigate the agents' incentives to free ride. In contrast to these papers, which highlight the inefficiencies that arise in these games and explore the differences to their static counterparts, our objective is to construct a mechanism that restores efficiency.

Our work is also related to a recent strand of the dynamic mechanism design literature that extends the Vickrey-Clarke-Groves and d'Aspremont-Gerard-Varet mechanisms to dynamic environments. Bergemann and Välimäki (2010) and Athey and Segal (2013) consider a dynamic model where in every period each agent obtains private information, and a social planner seeks to make allocation decisions based on the information that the agents report. After showing that a static mechanism cannot restore efficiency in this dynamic environment, they construct mechanisms that induce the agents to report truthfully and implement the efficient allocations after every history. The former paper focuses on the mechanism being self-enforcing, while the latter focuses on the mechanism being budget-balanced after every period. While these papers focus on environments with private information only, in an earlier working paper, Athey and Segal (2007) also incorporate moral hazard in the dynamic mechanism. The basic idea behind these mechanisms is that they make each agent a full residual claimant of total surplus, so the agent internalizes his externality on the other agents, which in turn induces him to report truthfully as long as the mechanism prescribes an efficient allocation rule. Our contribution relative to these papers is twofold. First, we focus on particular applications, which enables us to generate economic insights about the properties of the efficient mechanism in the context of those applications. Second, we extend the analysis to characterize mechanisms that condition on time in addition to the main state variable (e.g., progress on the project). This makes it possible to achieve efficiency even if the agents face tighter cash constraints.

Finally, there is a growing literature on contracting for experimentation such as Bergemann and Hege (2005); Bonatti and Hörner (2011); Halac, Kartik, and Liu (2015); Moroni (2014); and others. These papers consider a principal-agent problem in which the agents participate in a strategic experimentation game, and they characterize the optimal contract. In extending our model to dynamic experimentation problems, we approach this problem from a different perspective by characterizing a mechanism that induces the agents to always choose the efficient actions as part of an MPE. A secondary difference is that these papers typically assume that experimentation costs are linear and symmetric across the agents, whereas we allow for more general, asymmetric cost structures.

The remainder of this paper is organized as follows. We present the model in Section I; in Section II we characterize the MPE, as well as the efficient outcome of this game. In Section III, we present our main result: a budget-balanced mechanism 
that achieves efficiency as the outcome of an MPE. In Section IV, we extend our model to incorporate uncertainty, and we characterize the efficient (time-dependent) mechanism. In Section V, we adapt our mechanism to other dynamic games with externalities; and in Section VI, we conclude. All proofs are provided in the Appendix.

\section{Model}

A group of $n$ agents collaborate to complete a project. Time $t \in[0, \infty)$ is continuous. The project starts at initial state $q_{0}=0$, its state $q_{t}$ evolves at a rate that depends on the agents' instantaneous effort levels, and it is completed at the first time $\tau$ such that $q_{t}$ hits the completion state, denoted by $Q .{ }^{1}$ Each agent $i$ is risk-neutral, discounts time at rate $r>0$, has cash reserves $w_{i} \geq 0$ at the outset of the game, and receives a prespecified reward $V_{i}=\alpha_{i} V>0$ upon completing the project, where $\sum_{i=1}^{n} \alpha_{i}=1.2^{2}$ An incomplete project has zero value. At every moment $t$ each agent $i$ observes the state of the project $q_{t}$ and privately chooses his effort level $a_{i, t} \geq 0$, at flow cost $c_{i}\left(a_{i, t}\right)$, to influence the process

$$
\left.d q_{t}=\left(\sum_{i=1}^{n} a_{i, t}\right) d t\right]^{3}
$$

We assume that each agent's effort choice is not observable to the other agents, and $c_{i}(\cdot)$ is a strictly increasing, strictly convex, differentiable function satisfying $c_{i}^{\prime \prime \prime}(a) \geq 0$ for all $a, c_{i}(0)=c_{i}^{\prime}(0)=0$ and $\lim _{a \rightarrow \infty} c_{i}^{\prime}(a)=\infty$ for all $i$. Finally, we shall restrict attention to Markov perfect equilibria-henceforth, MPE-where at every moment each agent observes the current state of the project $q_{t}$ and chooses his effort level as a function of $q_{t}$, but not its entire evolution path $\left\{q_{s}\right\}_{s \leq t} \cdot{ }^{4}$

\section{Equilibrium Analysis}

For benchmarking purposes, in this section we characterize the MPE of this game without a mechanism in place, as well as the first-best outcome, i.e., the problem faced by a social planner who chooses the agents' strategies to maximize their total discounted payoff. Finally, we compare the MPE and the first-best outcome of the game to pinpoint the inefficiencies that arise in equilibrium.

\footnotetext{
${ }^{1}$ For simplicity, we assume that the completion state is specified exogenously. It is straightforward to extend the model to allow for endogenous project size and to show that all results carry over.

${ }^{2}$ Note that $V$ can be interpreted as the expected value of the project's random reward. We assume that the project generates a payoff only upon completion. It is straightforward to extend the model to the case in which the project also generates flow payoffs while it is in progress.

${ }^{3}$ We assume that the project progresses deterministically in the base model, because this makes it easier to illustrate the counteracting economic forces and to exposit the intuitions. We extend our model to incorporate stochastic progress in Section IV.

${ }^{4}$ We focus on the MPE, because it requires minimal coordination among the agents, and it constitutes the worst-case scenario in terms of the severity of the free-rider problem. As shown by Georgiadis, Lippman, and Tang (2014), this game has a continuum of non-Markovian equilibria that yield the agents a higher discounted payoff than the MPE.
} 


\section{A. Markov Perfect Equilibrium}

In an MPE, at every moment $t$ each agent $i$ observes the state of the project $q_{t}$ and chooses his effort $a_{i}\left(q_{t}\right)$ as a function of $q_{t}$ to maximize his discounted payoff while accounting for the effort strategies of the other group members $\left\{a_{j}\left(q_{t}\right)\right\}_{j \neq i}$. For a given set of strategies and a given initial value $q_{t}=q$, agent $i$ 's value function satisfies

$$
J_{i}(q)=e^{-r(\tau-t)} \alpha_{i} V-\int_{t}^{\tau} e^{-r(s-t)} c_{i}\left(a_{i}\left(q_{s}\right)\right) d s,
$$

where $\tau$ denotes the completion time of the project. Using standard arguments, we expect that the function $J_{i}(\cdot)$ satisfies the Hamilton-Jacobi-Bellman (henceforth, HJB) equation:

$$
r J_{i}(q)=\max _{a_{i}}\left\{-c_{i}\left(a_{i}\right)+\left(\sum_{j=1}^{n} a_{j}\right) J_{i}^{\prime}(q)\right\}
$$

defined on $[0, Q]$, subject to the boundary condition

$$
J_{i}(Q)=\alpha_{i} V
$$

The boundary condition states that upon completing the project, each agent receives his reward, and the game ends.

Assuming that (2) holds for all agents, each agent observes the state $q$, and chooses his effort to maximize the right side of (2) while accounting for his belief about the other agents' strategies $\left\{a_{j}(q)\right\}_{j \neq i}$. The corresponding first-order condition is $c_{i}^{\prime}\left(a_{i}\right)=J_{i}^{\prime}(q)$ : at every moment he chooses his effort level such that the marginal cost of effort is equal to the marginal benefit associated with bringing the project closer to completion. By noting that the second-order condition is satisfied, $c^{\prime}(0)=0$, and $c(\cdot)$ is strictly convex, it follows that for any $q$, agent $i$ 's optimal effort level is $a_{i}(q)=f_{i}\left(J_{i}^{\prime}(q)\right)$, where $f_{i}(\cdot)=c_{i}^{\prime-1}(\max \{0, \cdot\})$. By substituting this into (2), the discounted payoff function of agent $i$ satisfies

$$
r J_{i}(q)=-c_{i}\left(f_{i}\left(J_{i}^{\prime}(q)\right)\right)+\left[\sum_{j=1}^{n} f_{j}\left(J_{j}^{\prime}(q)\right)\right] J_{i}^{\prime}(q)
$$

subject to the boundary condition (3).

Thus, an MPE is characterized by the system of ordinary differential equations (henceforth, ODE) defined by (4) subject to the boundary condition (3) for all $i \in\{1, . ., n\}$, provided that this system admits a solution. The following proposition characterizes the unique project-completing MPE of this game and establishes its main properties. 
PROPOSITION 1: For $Q$ sufficiently small, the game defined by (2) subject to the boundary conditions (3) for all $i \in\{1, . ., n\}$ has an MPE that is unique among those in which each agent's discounted payoff is twice differentiable. The payoff is also strictly positive, strictly increasing, and strictly convex in $q$ on $[0, Q]$, i.e., $J_{i}(q)>0$, $J_{i}^{\prime}(q)>0$, and $J_{i}^{\prime \prime}(q)>0$. In particular, since each agent's optimal effort $a_{i}(q)$ $=f_{i}\left(J_{i}^{\prime}(q)\right)$, equilibrium effort is strictly increasing in $q$.

The first part of this proposition asserts that if the project is not too long (i.e., $Q$ is not too large), then the agents find it optimal to exert positive effort, in which case the project is completed in equilibrium. It is intuitive that $J_{i}(\cdot)$ is strictly increasing: each agent is better off the closer the project is to completion. That each agent's effort level increases with progress is also intuitive: since he incurs the cost of effort at the time effort is exerted but is only compensated upon completion of the project, his incentives are stronger the closer the project is to completion. An implication of this last result is that efforts are strategic complements across time. That is because by raising his effort an agent brings the project closer to completion, thus incentivizing agents to raise their future efforts (Kessing 2007).

It is noteworthy that the MPE need not be unique in this game. Unless the project is sufficiently short (i.e., $Q$ is sufficiently small) such that at least one agent is willing to undertake the project single-handedly, there exists another (Markov perfect) equilibrium in which no agent ever exerts any effort and the project is never completed. Finally, if the project is too long, then there exists no equilibrium in which any agent ever exerts positive effort.

\section{B. First-Best Outcome}

In this section, we consider the problem faced by a social planner, who at every moment chooses the agents' effort levels to maximize the group's total discounted payoff. Denoting the planner's discounted payoff function by $\bar{S}(q)$, we expect that it satisfies the HJB equation

$$
r \bar{S}(q)=\max _{a_{1}, . ., a_{n}}\left\{-\sum_{i=1}^{n} c_{i}\left(a_{i}\right)+\left(\sum_{i=1}^{n} a_{i}\right) \bar{S}^{\prime}(q)\right\}
$$

defined on interval $[0, Q]$, subject to the boundary condition

$$
\bar{S}(Q)=V
$$

The first-order conditions for the planner's problem are $c_{i}^{\prime}\left(a_{i}\right)=S^{\prime}(q)$ for every $i$ : at every moment each agent's effort is chosen such that the marginal cost of effort is equal to the group's total marginal benefit of bringing the project closer to completion. Note the difference between the MPE and the first-best outcome: here, each agent trades off his marginal cost of effort and the marginal benefit of progress to the entire group, whereas in the MPE, he trades off his marginal cost of effort and his marginal benefit of progress, ignoring the (positive) externality of his effort on the other agents. 
Denoting the first-best effort level of agent $i$ by $\bar{a}_{i}(q)$, we have $\bar{a}_{i}(q)=f_{i}\left(\bar{S}^{\prime}(q)\right)$, and by substituting the first-order condition into (5), it follows that the planner's discounted payoff function satisfies

$$
r \bar{S}(q)=-\sum_{i=1}^{n} c_{i}\left(f_{i}\left(\bar{S}^{\prime}(q)\right)\right)+\left[\sum_{i=1}^{n} f_{i}\left(\bar{S}^{\prime}(q)\right)\right] \bar{S}^{\prime}(q)
$$

subject to the boundary condition (6).

The following proposition characterizes the solution to the planner's problem.

PROPOSITION 2: For $Q$ sufficiently small, the ODE defined by (5) subject to the boundary condition 6) has a unique solution on $[0, Q]$. On this interval, the planner's discounted payoff is strictly positive, strictly increasing, twice differentiable, and strictly convex in $q$, i.e., $\bar{S}(q)>0, \bar{S}^{\prime}(q)>0$, and $\bar{S}^{\prime \prime}(q)>0$. Since the efficient level of effort for each agent $\bar{a}_{i}(q)=f_{i}\left(\bar{S}^{\prime}(q)\right)$, effort is strictly increasing in $q$.

Provided that the project is not too long (i.e., $Q$ is not too large), it is socially efficient to undertake and complete the project, whereas otherwise, the discounted cost to complete the project is less than the discounted reward that it generates, so it is efficient to not undertake it. The intuition for why each agent's effort level increases with progress is similar to that in Proposition 1. Throughout the remainder of this paper, we shall assume that $Q$ is sufficiently small such that it is socially efficient to undertake the project.

Because the project progresses deterministically, it is possible to study the completion time of the project, which is also deterministic. A useful comparative static for the analysis that follows concerns how the completion time $\tau$ depends on the group size $n$.

Remark 1: Suppose that the agents are identical, i.e., $c_{i}(a)=c_{j}(a)$ for all $i, j$, and $a$. Then the completion time of the project in the first-best outcome $\tau$ decreases in the group size $n$.

Intuitively, because effort costs are convex, the larger the number of agents in the group, the faster they will complete the project in the first-best outcome. As shown in Georgiadis (2015a), this is generally not true in the MPE, where the completion time of the project decreases in $n$ if and only if the project is sufficiently long (i.e., $Q$ is sufficiently large).

\section{Comparing the MPE and the First-Best Outcome}

Before we propose our mechanism, it is useful to pinpoint the inefficiencies that arise in this game when each agent chooses his effort to maximize his discounted payoff. To do so, we compare the solution to the planner's problem with the MPE in the following proposition. 
PROPOSITION 3: Suppose that the agents are symmetric (i.e., $\alpha_{i}=\alpha_{j}$ and $c_{i}(a)=c_{j}(a)$ for all $i, j$, and $\left.a \geq 0\right)$. Then in the MPE characterized in Proposition 1 , each agent's effort level $a_{i}(q) \leq \bar{a}_{i}(q)$ for all $q$, where $\bar{a}_{i}(q)$ is the first-best effort level characterized in Proposition 2.

Intuitively, because each agent's incentives are driven by the payoff that he receives upon completion of the project $\alpha_{i} V<V$, whereas the social planner's incentives are driven by the entire payoff that the project generates, in equilibrium, each agent exerts less effort relative to the first-best outcome, in the symmetric case. This result resembles the earlier results on free-riding in partnerships (e.g., Holmström 1982, Admati and Perry 1991, and others). 5

It turns out that in this dynamic game, there is a second, more subtle source of inefficiency. Because this game exhibits positive externalities and efforts are strategic complements across time, each agent has an incentive to front-load his effort relative to the first best in order to induce the other agents to raise their effort, which in turn renders him better off. ${ }^{6}$ More precisely, we have

PROPOSITION 4: For each agent $i$, the MPE discounted marginal cost of effort $e^{-r t} c_{i}^{\prime}\left(a_{i, t}\right)$ is decreasing in $t$, while it is equal to zero in the socially efficient outcome.

Therefore, each agent's discounted marginal cost of effort in the efficient outcome is constant over time. This is intuitive: because effort costs are convex, efficiency requires that the agents smooth their effort over time. On the other hand, in the MPE, each agent's discounted marginal cost of effort decreases over time. Intuitively, because at every moment $t$, each agent chooses his effort upon observing the state of the project $q_{t}$; his equilibrium effort level increases in $q$; and he is better off the harder others work. Each agent has incentives to front-load his effort in order to induce others to raise their future efforts.

\section{An Efficient Mechanism}

In this section, we establish our main result: we construct a mechanism that induces each self-interested agent to always exert the efficient level of effort when playing an MPE. This mechanism specifies upfront payments that are due at the outset (which turn out to be 0 in the optimal mechanism), flow payments that each agent must pay while the project is in progress, which are a function of $q$, and the reward that each agent receives upon completion of the project. We show that the mechanism is budget balanced on the equilibrium path, whereas off path, there may be a budget surplus, in which case money must be burned. However, the mechanism never results in a budget deficit.

\footnotetext{
${ }^{5}$ If the agents are asymmetric, then we can only establish a weaker result. The proof shows that for every $q$, there exists at least one agent $i$ such that his effort $a_{i}(q)$ is no greater than his first-best effort $\bar{a}_{i}(q)$.

${ }^{6}$ Front-loading here refers to an agent's discounted marginal cost of effort decreasing over time (and with progress), whereas efficiency requires that it be constant across time.
} 
The timing of the game is as follows: First, a mechanism is proposed, which specifies an upfront payment $P_{i, 0}$ for each agent $i$ to be made before work commences, and a schedule of flow payments $h_{i}\left(q_{t}\right) d t$ to be made during every interval $(t, t+d t)$ while the project is in progress. Then, each agent decides whether to accept or reject the mechanism. If all agents accept the mechanism, then each agent makes the upfront payment, and work commences. The upfront as well as the flow payments are placed in a savings account, which accumulates interest at rate $r$. If a payment that is due is not made, then the group dissolves, and each agent receives payoff zero.

An important remark is that if we interpret these flow payments as cash payments (as opposed to foregone salary, for example, as discussed in the introduction), then for this mechanism to be resource feasible, the agents must have sufficient cash reserves at the outset of the game (i.e., the $w_{i}$ s must be sufficiently large). We begin in Section IIIA by assuming that $w_{i}=\infty$ for all $i$ (i.e., that the agents have unlimited cash reserves), and we characterize a mechanism that implements the efficient outcome. In Section IIIB, we establish a necessary and sufficient condition for the mechanism to be resource feasible when the agents' cash reserves are limited. In Section IV, we extend our model to incorporate uncertainty in the evolution of the project, and we consider a broader class of mechanisms, which depend on both time $t$ and the state $q \cdot{ }^{7}$

\section{A. Unlimited Cash Reserves}

Incentivizing Efficient Actions. - Given a set of flow-payment functions $\left\{h_{i}(q)\right\}_{i=1}^{n}$, agent $i$ 's discounted payoff function (excluding the upfront payments $P_{i, 0}$ ), which we denote by $\hat{J}_{i}(q)$, satisfies the HJB equation

$$
r \hat{J}_{i}(q)=\max _{a_{i}}\left\{-c_{i}\left(a_{i}\right)+\left(\sum_{j=1}^{n} a_{j}\right) \hat{J}_{i}^{\prime}(q)-h_{i}(q)\right\}
$$

on $[0, Q]$, subject to a boundary condition that remains to be determined. His first-order condition is $c_{i}^{\prime}\left(a_{i}\right)=\hat{J}_{i}^{\prime}(q)$, and because we want to induce each agent to exert the efficient effort level, we must have $c_{i}^{\prime}\left(a_{i}\right)=\bar{S}^{\prime}(q)$, where $\bar{S}(\cdot)$ is characterized in Proposition 2. Therefore,

$$
\hat{J}_{i}(q)=\bar{S}(q)-p_{i}
$$

where $p_{i}$ is a constant to be determined. Upon completion of the project, agent $i$ must receive

$$
\hat{J}_{i}(Q)=\bar{S}(Q)-p_{i}=V-p_{i}
$$

\footnotetext{
${ }^{7}$ In the deterministic case, we characterize a time-dependent mechanism that induces efficient incentives along the equilibrium path even if the agents have no cash reserves (i.e., $w_{i}=0$ for all $i$ ).
} 
which gives us the boundary condition. Note that the upfront payments $\left\{P_{i, 0}\right\}_{i=1}^{n}$ and the constants $\left\{p_{i}\right\}_{i=1}^{n}$ act as transfers, and we will choose them to minimize the total cash reserves needed to implement the mechanism, and to ensure that the mechanism is budget balanced and the agents' individual rationality constraints are satisfied (i.e., each agent's ex ante discounted payoff is nonnegative). Using (7) and (8), we can solve for each agent's flow-payment function that induces first-best effort:

$$
h_{i}(q)=\sum_{j \neq i} c_{j}\left(f_{j}\left(\bar{S}^{\prime}(q)\right)\right)+r p_{i} .
$$

Based on this analysis, we can establish the following lemma.

LEMMA 1: Suppose that all agents make flow payments that satisfy (9) and receive $V-p_{i}$ upon completion of the project. Then:

(i) There exists an MPE in which at every moment each agent exerts the efficient level of effort $\bar{a}_{i}(q)$ as characterized in Proposition 2.

(ii) The efficient flow-payment function satisfies $h_{i}^{\prime}(q)>0$ for all i and $q \in[0, Q]$, i.e., the flow payments are strictly increasing in the state of the project.

To understand the intuition behind this construction, recall that the MPE characterized in Section IIA is plagued by two sources of inefficiency: first, the agents have incentives to shirk by exerting inefficiently low effort, and second, they front-load their effort, i.e., their discounted marginal cost of effort decreases over time, whereas it is constant in the efficient outcome. Therefore, to induce agents to always exert the first-best effort, this mechanism must neutralize both sources of inefficiency. To eradicate the former, each agent is effectively made the full residual claimant of the project. ${ }^{8}$ To neutralize the latter, the flow payments increase with progress at a rate such that each agent's benefit from front-loading effort is exactly offset by the cost associated with having to make larger flow payments in the future.

By observing (9), we make the following remark regarding the total flow cost that each agent incurs while the project is in progress.

Remark 2: The flow cost that agent $i$ incurs given the state of the project $q$ is equal to

$$
c_{i}\left(f_{i}\left(\bar{S}^{\prime}(q)\right)\right)+h_{i}(q)=\sum_{j=1}^{n} c_{j}\left(f_{j}\left(\bar{S}^{\prime}(q)\right)\right)+r p_{i},
$$

i.e., it is the same across all agents and equal to the flow cost faced by the social planner (up to a constant).

\footnotetext{
${ }^{8}$ Notice that each agent $i$ receives $V-p_{i}$ upon completion of the project, and his ad infinitum discounted cost of the state-independent flow payments in (9) is equal to $\int_{0}^{\infty} e^{-r t} r p_{i} d t=p_{i}$. Therefore, the net benefit to each agent from completing the project is equal to $\left(V-p_{i}\right)-\left(-p_{i}\right)=V$; hence, he effectively becomes a residual claimant.
} 
Intuitively, to induce efficient incentives, the flow-payment function for each agent $i$ must be chosen such that he faces the same flow cost (and reward upon completion) that the social planner faces in the original problem (up to a constant).

Budget Balance.-In this section, we characterize the payments $\left\{P_{i, 0}\right\}_{i=1}^{n}$ and $\left\{p_{i}\right\}_{i=1}^{n}$ such that the mechanism is budget balanced while at the same time minimizing the total discounted cost of the upfront and flow payments along the efficient effort path.

We will say that a mechanism is budget balanced if the total sum of the discounted payments is equal to the total reward generated by the project, i.e.,

$$
\sum_{i=1}^{n}\left[P_{i, 0}+\int_{0}^{\tau} e^{-r s} h_{i}\left(q_{s}\right) d s+e^{-r \tau}\left(V-p_{i}\right)\right]=e^{-r \tau} V .
$$

Before the agents begin working on the project, each agent $i$ 's equilibrium discounted payoff is equal to $\hat{J}_{i}(0)-P_{i, 0}=\bar{S}(0)-p_{i}-P_{i, 0}$. Because each agent exerts the efficient effort level along the equilibrium path, the sum of the agents' ex ante discounted payoffs (before any upfront payments) must equal $\bar{S}(0)$. Therefore, budget balance requires that $n \bar{S}(0)-\sum_{i=1}^{n}\left(p_{i}+P_{i, 0}\right)=\bar{S}(0)$, or equivalently, $\sum_{i=1}^{n}\left(P_{i, 0}+p_{i}\right)=(n-1) \bar{S}(0)$. Notice that the payments $\left\{P_{i, 0}\right\}_{i=1}^{n}$ and $\left\{p_{i}\right\}_{i=1}^{n}$ are not determined uniquely.

The total discounted cost of the payments along the equilibrium path is equal to

$$
\sum_{i=1}^{n}\left[P_{i, 0}+\int_{0}^{\bar{\tau}} e^{-r s}\left(\sum_{j \neq i} c_{j}\left(f_{j}\left(\bar{S}^{\prime}\left(q_{s}\right)\right)\right)+r p_{i}\right) d s\right]=e^{-r \bar{\tau}}\left[(n-1) V-\sum_{i=1}^{n} p_{i}\right],
$$

where $\bar{\tau}$ denotes the first-best completion time. A necessary condition for the mechanism to never be in deficit is $\sum_{i=1}^{n} P_{i, 0} \geq 0$, so $\sum_{i=1}^{n} p_{i} \leq(n-1) \bar{S}(0)$. Moreover, notice that the total discounted cost of the payments is minimized when

$$
\sum_{i=1}^{n} p_{i}=(n-1) \bar{S}(0) \text { and } P_{i, 0}=0 \forall i
$$

Accordingly, from now on, we set $P_{i, 0}=0$, i.e., all upfront payments are set to 0 . Notice that the budget-balance constraint pins down only the sum $\sum_{i=1}^{n} p_{i}$, while the individual $p_{i} \mathrm{~s}$ will depend on the way the agents decide to share the profits. Furthermore, observe from (9) that the sum of the agents' flow payments is always nonnegative, which together with the above conditions implies that the mechanism is never in deficit along the equilibrium path.

So far, we have characterized a mechanism that induces each agent to exert the first-best effort at every moment while the project is in progress and that is budget balanced in equilibrium. However, a potential concern is that following a deviation, the total amount in the savings account upon completion of the project may be greater or less than $(n-1)[V-\bar{S}(0)]$, in which case the mechanism will have a budget surplus or deficit, respectively. A desirable attribute is for the mechanism to always be budget balanced, so that the rewards that the agents receive upon 
completion of the project sum up to the balance in the savings account, both on and off the equilibrium path. To analyze this case, we let

$$
H_{t}=\sum_{i=1}^{n} \int_{0}^{t} e^{r(t-s)} h_{i}\left(q_{s}\right) d s
$$

denote the balance in the savings account at time $t$. We say that the mechanism is strictly budget balanced if the sum of the agents' rewards upon completion of the project (at time $\tau$ ) is equal to $V+H_{\tau}$. We assume that each agent $i$ is promised reward $\beta_{i}\left(V+H_{\tau}\right)$ upon completion of the project, where $\beta_{i} \in[0,1]$ for all $i$ and $\sum_{i=1}^{n} \beta_{i}=1$. The following lemma shows that there exists no efficient, strictly budget-balanced mechanism.

LEMMA 2: Suppose that each agent i receives $\beta_{i}\left(V+H_{\tau}\right)$ upon completion, where $\beta_{i} \in[0,1]$ for all $i$ and $\sum_{i=1}^{n} \beta_{i}=1$. Then there exist no flow-payment functions $h_{i}(\cdot)$ that lead to the efficient outcome in an MPE. In particular, the maximal social welfare of the strictly budget-balanced game is equal to the maximal social welfare of a game in which each player $i$ is promised reward $\beta_{i} V$ upon completion of the project, and the flow payments add up to zero, i.e., $\sum_{i=1}^{n} h_{i}(q)=0$ for all $q$.

An implication of this result is that it is impossible to construct a mechanism that is simultaneously efficient and strictly budget balanced. Moreover, if we require flow-payment functions $h_{i}(\cdot)$ to be nonnegative (or if we restrict attention to symmetric agents and symmetric mechanisms), then there exists no strictly budget-balanced mechanism that yields a greater total discounted payoff as an outcome of an MPE than the payoff corresponding to the case characterized in Proposition 1.

While this is a negative result, as shown in the following proposition, it is possible to construct a mechanism that is efficient and budget balanced on the equilibrium path (but not strictly budget balanced) and which will never have a budget deficit.

PROPOSITION 5: Suppose that each agent commits to making flow payments as given by (9) for all $q$, where $\sum_{i=1}^{n} p_{i}=(n-1) \bar{S}(0)$, and he receives lumpsum reward $\min \left\{V-p_{i}, \beta_{i}\left(V+H_{\tau}\right)\right\}$ upon completion of the project, where $\beta_{i}=\frac{V-p_{i}}{V+(n-1)[V-\bar{S}(0)]}$. Then:

(i) There exists a Markov-perfect equilibrium in which at every moment each agent exerts the efficient level of effort $\bar{a}_{i}(q)$ as characterized in Proposition 2.

(ii) The mechanism is budget balanced on the equilibrium path, and it will never result in a budget deficit (including off equilibrium).

(iii) The agents'total ex ante discounted payoff is equal to the first-best discounted payoff, i.e., $\sum_{i=1}^{n} \hat{J}_{i}(0)=\bar{S}(0)$. 
The intuition for why there exists no efficient, strictly budget-balanced mechanism (as shown in Lemma 2) is as follows: to attain efficiency, the mechanism must eliminate the agents' incentives to shirk. However, with strict budget balance, by shirking, an agent can delay the completion of the project, let the balance in the savings account grow, and collect a larger reward upon completion. By capping each agent's reward to $V-p_{i}$, his incentive to procrastinate is eliminated, and efficiency becomes attainable.

Note that because the mechanism may burn money off the equilibrium path, it is important that the agents commit to not renegotiating the mechanism should that contingency arise. 9

\section{B. Limited (but Sufficient) Cash Reserves}

Because the mechanism requires that the agents make flow payments while the project is in progress, two complications arise when they have limited cash reserves. First, the mechanism must incorporate the possibility that one or more agents run out of cash before the project is completed. Second, for the efficient mechanism to be resource feasible, the agents must have sufficient cash in hand. This section is concerned with addressing these issues.

The following proposition establishes a necessary and sufficient condition for the mechanism characterized in Proposition 5 to be resource feasible; that is, each agent has enough cash $w_{i}$ to make the flow payments $h_{i}(\cdot)$ on the equilibrium path.

PROPOSITION 6: The mechanism characterized in Proposition 5 is resource feasible if and only if there exist $\left\{p_{i}\right\}_{i=1}^{n}$ such that $\sum_{i=1}^{n} p_{i}=(n-1) \bar{S}(0)$,

$$
p_{i}\left(1-e^{-r \bar{\tau}}\right) \leq w_{i}-\int_{0}^{\bar{\tau}} e^{-r t} \sum_{j \neq i} c_{j}\left(f_{j}\left(\bar{S}^{\prime}\left(q_{t}\right)\right)\right) d t \text { and } p_{i} \leq \bar{S}(0)
$$

for all $i$, where $\bar{S}(\cdot)$ is characterized in Proposition 2, and $\bar{\tau}$ is the corresponding first-best completion time.

If the agents are symmetric $\left(\right.$ i.e., $c_{i}(\cdot) \equiv c_{j}(\cdot)$ and $w_{i}=w_{j}$ for all $i$ and $\left.j\right)$, then the mechanism is resource feasible if and only if $w_{i} \geq e^{-r \bar{\tau}}\left(\frac{n-1}{n}\right)[V-\bar{S}(0)]$ for all $i$, where this threshold increases in the group size $n .10$

The first condition asserts that each agent must have sufficient cash to make the flow payments $h_{i}(\cdot)$ on the equilibrium path. The second condition is each agent's individual rationality constraint, i.e., each agent's ex ante discounted payoff must be

\footnotetext{
${ }^{9}$ This contingency will arise if the agents exert less than first-best effort, which will result in the project being completed at some time $\tau>\bar{\tau}$ and the balance in the savings account $H_{\tau}$ exceeding $(n-1) V$. In this case, the mechanism specifies that each agent $i$ receives reward $V-p_{i}$ upon completion and the surplus $H_{\tau}-(n-1) V$ is burned.

${ }^{10}$ This follows from Remark 1, which shows that the first-best completion time $\bar{\tau}$ decreases in $n$. Therefore, $e^{-r \bar{\tau}}\left(\frac{n-1}{n}\right)\left[V-\bar{S}\left(q_{0}\right)\right]$ increases in $n$.
} 
nonnegative. In general, these conditions are satisfied if each agent's cash reserves are sufficiently large. ${ }^{11,12}$

Intuitively, the minimum cash reserves needed to implement the mechanism increase in the group size, because the inefficiencies due to shirking and front-loading become more severe the larger the group. As a result, the mechanism must specify larger payments to neutralize those inefficiencies.

Even if the conditions in Proposition 6 are satisfied, it is still possible, off equilibrium, for one or more agents to run out of cash before the project is completed, in which case they will be unable to make the flow payments specified by the mechanism. Thus, the mechanism must specify the flow payments as a function not only of the state $q$, but also of the agents' cash reserves.

To analyze this case, we denote by $I_{i}(q)$ the indicator random variable that is equal to one if agent $i$ has not run out of cash at any state $\tilde{q}<q$ and zero otherwise. The following proposition shows that the efficiency properties established in Proposition 5 continue to hold if each agent "loses" his share when he runs out of cash before the project is completed.

PROPOSITION 7: Suppose each agent commits to making flow payments $h_{i}(q) I_{i}(q)$ for all $q$ (where $\left.\sum_{i=1}^{n} p_{i}=(n-1) \bar{S}(0)\right)$, and upon completion of the project, he receives lump-sum reward $\min \left\{V-p_{i}, \beta_{i}\left(V+H_{\tau}\right)\right\} I_{i}(Q)$, where $\beta_{i}=\frac{V-p_{i}}{V+(n-1)[V-\bar{S}(0)]}$. Moreover, assume the conditions of Proposition 6 are satisfied. Then the properties of Proposition 5 continue to hold.

For the mechanism to induce efficient incentives, it must punish any agent who runs out of cash, for this should not occur in equilibrium. In addition, if an agent runs out of cash, then the share that he "loses" must be burned, i.e., it cannot be shared among other agents. This is to deter agents from shirking as a means of running an agent out of cash in order to collect his "lost" share.

\section{Uncertainty and Time Dependence}

To simplify the exposition and distill the economic intuition, we have assumed that the project progresses deterministically and restricted attention to the set of mechanisms that depend on the state of the project $q$, but not on time $t$. In this section, we relax these restrictions by using a model similar to that in Georgiadis

\footnotetext{
${ }^{11}$ Note that in the symmetric case, we have $p_{i}=\left(\frac{n-1}{n}\right) \bar{S}(0)$ and $\int_{0}^{\bar{\tau}} e^{-r t} \sum_{j \neq i} c_{j}\left(f_{j}\left(\bar{S}^{\prime}\left(q_{t}\right)\right)\right)$ $=\frac{n-1}{n}\left[e^{-r \bar{\tau}} V-\bar{S}(0)\right]$ for all $i$. Thus, the first condition reduces to $w_{i}>e^{-r \bar{\tau}}\left(\frac{n-1}{n}\right)[V-\bar{S}(0)]$ and the second condition is automatically satisfied.

${ }^{12}$ We have also characterized the time-independent mechanism that maximizes the agents' discounted payoff when the conditions established in Section IIIB are not satisfied, and hence the efficient mechanism cannot be implemented. This analysis is available upon request. However, we show below that the efficient mechanism can be implemented when allowing time dependence in such a way that the flow payments are equal to zero on the equilibrium path.
} 
(2015a), and we characterize the efficient, time-dependent mechanism in an uncertain environment. In this model, the state of the project progresses according to

$$
d q_{t}=\left(\sum_{i=1}^{n} a_{i, t}\right) d t+\sigma d W_{t}
$$

where $W_{t}$ is a standard Brownian motion and $\sigma>0$ captures the degree of uncertainty associated with the evolution of the project. Moreover, we assume throughout that $w_{i}=\infty$ for all $i$ (i.e., that agents have unlimited cash reserves). The model is otherwise identical to the one presented in Section I. The planner's problem satisfies the ODE

$$
r \bar{S}(q)=-\sum_{i=1}^{n} c_{i}\left(f_{i}\left(\bar{S}^{\prime}(q)\right)\right)+\left[\sum_{i=1}^{n} f_{i}\left(\bar{S}^{\prime}(q)\right)\right] \bar{S}^{\prime}(q)+\frac{\sigma^{2}}{2} \bar{S}^{\prime \prime}(q)
$$

subject to the boundary conditions

$$
\lim _{q \rightarrow-\infty} \bar{S}(q)=0 \text { and } \bar{S}(Q)=V .
$$

Georgiadis (2015a) provides conditions under which (11) subject to (12) admits a unique, smooth solution. ${ }^{13}$ Each agent's first-best level of effort satisfies $\bar{a}_{i}(q)$ $=f_{i}\left(\bar{S}^{\prime}(q)\right)$, and (similarly to the deterministic case) $\bar{a}_{i}^{\prime}(q)>0$ for all $i$ and $q$.

Next consider each agent's problem facing an arbitrary flow-payments function $h_{i}(t, q)$ and a reward function $g_{i}(t)$. Using standard arguments, we expect agent $i$ 's discounted payoff function to satisfy the HJB equation

$$
r J_{i}(t, q)-J_{t, i}(t, q)=\max _{a_{i}}\left\{-c_{i}\left(a_{i}\right)+\left(\sum_{j=1}^{n} a_{j}\right) J_{q, i}(t, q)+\frac{\sigma^{2}}{2} J_{q q, i}(t, q)-h_{i}(t, q)\right\}
$$

subject to $\lim _{q \rightarrow-\infty} \hat{J}_{i}(q)=0$ and $J_{i}(t, Q)=g_{i}(t) .{ }^{14}$ His first-order condition is $c_{i}^{\prime}\left(a_{i}\right)=J_{q, i}(t, q)$, and because we want to induce each agent to exert the efficient effort level, we must have $c_{i}^{\prime}\left(a_{i}\right)=\bar{S}^{\prime}(q)$. Therefore, it needs to be the case that each agent's discounted payoff satisfy

$$
J_{i}(t, q)=\bar{S}(q)-p_{i}(t)
$$

where $p_{i}(\cdot)$ is a function of time that remains to be determined. From the boundary conditions for $J_{i}(\cdot, \cdot)$ and $\bar{S}(\cdot)$, it follows that $p_{i}(t)=V-g_{i}(t)$, and using this, together with (13) and (11), we can see that the flow-payment function that induces first-best effort is

$$
h_{i}(t, q)=\sum_{j \neq i} c_{j}\left(f_{j}\left(\bar{S}^{\prime}(q)\right)\right)+r\left[V-g_{i}(t)\right]+g_{i}^{\prime}(t) .
$$

\footnotetext{
${ }^{13}$ Roughly, a sufficient condition is that each agent's effort cost function $c_{i}(\cdot)$ is sufficiently convex (i.e., $c_{i}^{\prime}(a)$ is sufficiently large for large values of $a$ ). This condition is satisfied if, for example, $c_{i}(a)=\lambda_{i} a^{p}$, where $p \geq 2$.

${ }^{14} J_{t, i}(t, q)$ and $J_{q, i}(t, q)$ denote the derivative of $J_{i}(t, q)$ with respect to $t$ and $q$, respectively.
} 
We choose the set of functions $\left\{g_{i}(t)\right\}_{i=1}^{n}$ such that along the equilibrium (or equivalently, the first-best) path $\bar{q}_{t}$, each agent $i$ 's expected flow payment $E\left[h\left(t, \bar{q}_{t}\right)\right]$ is equal to zero, i.e.,

$$
0=\sum_{j \neq i} E\left[c_{j}\left(f_{j}\left(\bar{S}^{\prime}\left(\bar{q}_{t}\right)\right)\right)\right]+r\left(V-g_{i}(t)\right)+g_{i}^{\prime}(t)
$$

where the expectation is taken with respect to the state of the project along the equilibrium path. This is an ODE that can be solved for any given initial condition $g_{i}(0) \leq V$. To ensure that the sum of the agents' discounted payoffs is equal to the first-best payoff, the set of initial conditions $\left\{g_{i}(0)\right\}_{i=1}^{n}$ must satisfy $\sum_{i=1}^{n} J_{i}(0,0)$ $=\bar{S}(0)$, or equivalently,

$$
\sum_{i=1}^{n} g_{i}(0)=V+(n-1)[V-\bar{S}(0)]
$$

By substituting (14) into $h_{i}(t, q)$, we get the following expression for each agent's flow-payment function that induces first-best efforts:

$$
h_{i}(t, q)=\sum_{j \neq i}\left[c_{j}\left(f_{j}\left(\bar{S}^{\prime}(q)\right)\right)-E\left[c_{j}\left(f_{j}\left(\bar{S}^{\prime}\left(\bar{q}_{t}\right)\right)\right)\right]\right] .
$$

Finally, it follows from (16) that (15) ensures that the mechanism is ex ante budget balanced (in expectation). ${ }^{15}$ Therefore, we have established the following proposition:

PROPOSITION 8: Suppose that each agent makes flow payments $h_{i}(t, q)$ and receives $g_{i}(\tau)$ upon completion of the project, where $h_{i}(t, q)$ and $g_{i}(\tau)$ satisfy $(16)$ and (14), respectively. Then, $g_{i}(\tau)$ decreases in $\tau$, and there exists an MPE in which at every moment each agent exerts the first-best effort level. In this equilibrium, the sum of the agents' expected discounted payoffs is equal to the first-best expected discounted payoff, and each agent's local flow payment has expected value equal to 0. Moreover, this mechanism is ex ante budget balanced (i.e., in expectation).

The key difference relative to the time-independent case is that the mechanism now has two levers at its disposal to neutralize the inefficiencies described in Section IIC. To neutralize the agents' incentives to front-load effort, the mechanism specifies that flow payments are positive if and only if the current state $q$ is strictly greater than $E\left[\bar{q}_{t}\right]$, i.e., the expected state if all agents were exerting the efficient level of effort. To eliminate the agents' incentives to shirk due to receiving only

\footnotetext{
${ }^{15}$ Noting that the ex ante budget-balance condition is $\sum_{i=1}^{n} E\left[\int_{0}^{\tau} e^{-r t} h_{i, t} d t-e^{-r \tau} g_{i}(\tau)+e^{-r \tau V} \frac{V}{n}\right]=0$, and by using (1) and (16), one can show that it is equivalent to (15).
} 
a share of the project's payoff, the mechanism specifies that each agent's reward decreases in the completion time $\tau$ at a sufficiently rapid rate. 16

There are two important differences relative to the deterministic mechanism. First, in the stochastic mechanism, it is impossible to simultaneously achieve efficiency and ex post budget balance. Instead, the mechanism is budget balanced only in expectation, and as a result, for the mechanism to be resource feasible, there needs to be a third party who collects the surplus if the balance in the savings account upon completion of the project exceeds $\sum_{i=1}^{n} g_{i}(\tau)-V$ and who pays the difference otherwise. Second, flow payments may be positive or negative, and because the path of the Brownian motion until the project is completed can be arbitrarily long, for the mechanism to be resource feasible, it is necessary that the agents as well as the third party be cash unconstrained. 17

The following remark characterizes the set of time-independent mechanisms that implement the efficient outcome in the game with uncertainty.

Remark 3: Let $g_{i}(t)=G_{i}$, where $G_{i} \geq 0$ and $\sum_{i=1}^{n} G_{i}=V+(n-1)[V-\bar{S}(0)]$. Suppose that each agent makes (time-independent) flow payments that satisfy (16) and receives $G_{i}$ upon completion of the project. Then, there exists an MPE in which at every moment each agent exerts the first-best effort level. Moreover, this mechanism is ex ante budget balanced (i.e., in expectation).

Special Case: $\sigma=0$.-We now characterize the efficient time-dependent mechanism when the project progresses deterministically, i.e., when $\sigma=0$. The analysis is the same as in the stochastic case, except that the efficient path $\bar{q}_{t}$ is now deterministic. As a result, the efficient mechanism specifies zero flow payments on the equilibrium path (and positive flow payments only off equilibrium).

COROLLARY 1: Suppose that eachagent makes flow payments $\hat{h}_{i}(t, q)=\left[h_{i}(t, q)\right]^{+}$ and receives $\hat{g}_{i}(\tau)=\left[\min \left\{\alpha_{i} V, g_{i}(\tau)\right\}\right]^{+}$upon completion of the project, which decreases in $\tau$, where $h_{i}(t, q)$ and $g_{i}(\tau)$ satisfy (16) and (14), respectively. Then there exists an MPE in which at every moment each agent exerts the first-best effort level, makes 0 flow payments along the evolution path of the project, and receives reward $\alpha_{i} V$ upon completion. In this equilibrium, the sum of the agents'discounted payoffs is equal to the first-best discounted payoff. Moreover, this mechanism is budget balanced on the equilibrium path and never has a budget deficit.

By specifying a reward $g_{i}(\tau)$ that decreases in the completion time $\tau$, the mechanism induces each agent to complete the project by no later than the efficient completion time $\bar{\tau}$. To neutralize the agents' incentives to front-load effort, the mechanism specifies that as long as the state $q$ at time $t$ coincides with $\bar{q}(t)$, the flow payments

\footnotetext{
${ }^{16}$ This is reminiscent of the optimal contract in Mason and Välimäki (2015) who study "Poisson projects."

${ }^{17}$ However, this is less of an issue if one interprets the flow payments as difference between the entrepreneur's wage in the startup and the market rate if he were to seek employment elsewhere, as discussed in the introduction.
} 
are 0 , but they are strictly positive whenever $q>\bar{q}(t)$, which will occur if one or more agents work harder than first best.

\section{Other Applications}

We have developed our efficient mechanism in the context of games of dynamic contributions to a public good. More broadly however, our mechanism can be readily applied to any dynamic game with externalities. In this section, we illustrate this versatility by extending our approach to two classes of games that have been extensively studied in the economics literature - a dynamic common resource extraction problem and a strategic experimentation problem - and we construct a mechanism, which at every moment induces the agents to choose the efficient actions in an MPE. To simplify the exposition, we shall assume throughout this section that each agent has large cash reserves at the outset of the game, and we will restrict attention to the set of time-independent mechanisms.

\section{A. Dynamic Extraction of an Exhaustible Common Resource}

First, we adapt our mechanism to a game in which a group of agents extracts an exhaustible common resource over time, similar to the one studied by Levhari and Mirman (1980) and Reinganum and Stokey (1985).

Model.-A group of $n$ agents jointly extracts a common resource. Time $t \in[0, \infty)$ is continuous. The initial stock of the resource at time 0 is equal to $Q$, and it decreases according to

$$
d q_{t}=-\sum_{i=1}^{n} a_{i, t} d t
$$

where $a_{i, t} \geq 0$ denotes the instantaneous extraction rate of agent $i$ at time $t$. At every moment $t$, each agent $i$ observes the remaining stock $q_{t}$, and chooses his extraction rate $a_{i, t}$, which yields him flow utility $u_{i}\left(a_{i, t}\right)$, where $u_{i}(\cdot)$ is a strictly increasing, strictly concave function satisfying $u_{i}(0)=0, u_{i}^{\prime}(0)>0$, and $\lim _{c \rightarrow \infty} u_{i}^{\prime}(c)=0$. The game ends at the first stopping time $\tau$ such that $q_{\tau}=0$, i.e., as soon as the resource is depleted.

Efficient Mechanism.-We begin by considering the problem faced by a social planner who chooses the agents' extraction rates to maximize the discounted total surplus. His discounted payoff function satisfies the HJB equation

$$
r \bar{S}(q)=\max _{a_{1}, ., a_{n}}\left\{\sum_{i=1}^{n} u_{i}\left(a_{i}\right)-\left(\sum_{i=1}^{n} a_{i, t}\right) \bar{S}^{\prime}(q)\right\}
$$

subject to the boundary condition $\bar{S}(0)=0$. This condition states that the game ends and the agents receive no further payoffs as soon as the resource is depleted. The corresponding first-order conditions are $u_{i}^{\prime}\left(a_{i}\right)=\bar{S}^{\prime}(q)$ for all $i$, i.e., each 
agent's marginal utility from extraction must at all times be equal to the marginal cost associated with a smaller amount of the resource remaining in the future. Under regularity conditions on $u_{i}(\cdot)$, a solution to this problem exists, and we shall denote each agent's efficient extraction rate by $\bar{a}_{i}(q) .{ }^{18}$ It is straightforward to show that along the efficient extraction path, each agent extracts the resource at a slower rate as it becomes more scarce; in other words, $\bar{a}_{i}(q)$ increases in $q$.

We consider the set of mechanisms that specify flow subsidies as a function of the remaining amount of the resource $q$, which we denote by $\left\{s_{i}(q)\right\}_{i=1}^{n}$. Given an arbitrary mechanism, each agent $i$ 's discounted payoff satisfies the HJB equation

$$
r J_{i}(q)=\max _{a_{i}}\left\{u_{i}\left(a_{i}\right)-\left(\sum_{i=1}^{n} a_{i, t}\right) J_{i}^{\prime}(q)+s_{i}(q)\right\}
$$

subject to a boundary condition that remains to be determined. Each agent's first-order condition is now $u_{i}^{\prime}\left(a_{i}\right)=J_{i}^{\prime}(q)$, so to implement the first-best actions, the mechanism must be such that $\hat{J}_{i}^{\prime}(q)=\bar{S}^{\prime}(q)$, which implies that $J_{i}(q)=\bar{S}(q)-p_{i}$ for all $i$ and $q$, where $p_{i}$ is a constant to be determined. Notice that $J_{i}(0)=-p_{i}$, i.e., as soon as the resource is depleted, each agent $i$ must pay a penalty $p_{i}$ to the social planner and the game ends. By using (17) and solving for $s_{i}(q)$ in (18), one obtains

$$
s_{i}(q)=\sum_{j \neq i} u_{j}\left(\bar{a}_{j}(q)\right)-r p_{i} .
$$

Observe that the mechanism pays each agent a flow subsidy that decreases as the resource becomes more scarce (i.e., $s_{i}^{\prime}(q) \geq 0$ for all $i$ and $q$ ). Moreover, if $p_{i}>0$, then the flow subsidy can become negative for sufficiently small values of the stock. Intuitively, because in equilibrium the agents do not internalize the negative externality of their actions on the other agents, they have incentives to overharvest the resource. To counteract their incentives to free ride and to restore efficiency, the mechanism specifies subsidies that decrease in the remaining stock at a rate such that the cost associated with receiving a smaller subsidy (or having to make a larger payment) in the future exactly offsets each agent's present benefit from overharvesting the resource.

Next, suppose that the social planner requires that the budget be balanced (in equilibrium). Allowing upfront payments, which we denote by $\left\{P_{i, 0}\right\}_{i=1}^{n}$, the sum of the agents' ex ante discounted payoffs must be equal to the planner's discounted payoff, so

$$
\begin{aligned}
\sum_{i=1}^{n}\left[J_{i}(Q)-p_{i}-P_{i, 0}\right] & =n S(Q)-\sum_{i=1}^{n}\left(p_{i}+P_{i, 0}\right) \\
& =S(Q) \Rightarrow \sum_{i=1}^{n}\left(p_{i}+P_{i, 0}\right)=(n-1) S(Q) .
\end{aligned}
$$

\footnotetext{
${ }^{18}$ For example, if $u_{i}(a)=\lambda a^{\rho}$, where $\lambda>0$ and $\rho \in(0,1)$, then it is possible to characterize the solution to the planner's problem analytically.
} 
In summary, the efficient mechanism specifies that each agent must pay an "entry" fee $P_{i, 0}$ in exchange for access to the resource, receives a subsidy $s_{i}(q)$ that is a function of the amount of resource that remains, and must pay a penalty $p_{i}$ at the moment the resource is depleted. Notice that the budget-balance condition only pins down the sum of the entry fees and the penalties due upon depletion. The individual amounts will depend on the parties' cash constraints and the way they decide to split the profits. For example, if the agents are cash constrained, then the planner might set $P_{i, 0}=0$ for all $i$ and choose the penalties such that $\sum_{i=1}^{n} p_{i}$ $=(n-1) S(Q)$, which the agents will be able to fund using the subsidies that they receive. In this case, the budget is balanced, and provided that the agents cannot default on the penalty payments, the mechanism can be implemented even if the agents are cash constrained. On the other hand, if the social planner is cash constrained, then she might set $p_{i}=0$ for all $i$ and choose the entry fees such that $\sum_{i=1}^{n} P_{i, 0}=(n-1) S(Q)$.

\section{B. Strategic Experimentation}

We now consider a strategic experimentation game similar to the ones studied by Keller, Rady, and Cripps (2005) and Bonatti and Hörner (2011), and we construct a mechanism that restores efficiency in an MPE.

Model.-A group of $n$ agents faces a two-armed bandit problem, and each agent continuously chooses how much (costly) effort to allocate to each arm. The first arm is "safe:" it yields an expected flow payoff that is equal to 0 (net of the cost of effort) and known to all players. The other arm is "risky," and it can be either "bad" or "good" for all players. If it is bad, then it never yields any payoff. If it is good, then it yields a lump-sum payoff equal to 1 at random times that arrive according to a Poisson process with rate equal to each agent's instantaneous effort level. The arrival of these lump sums is independent across the agents (conditional on the arm being good). The agents hold a common prior belief $q_{0}$ about the probability that the risky arm is good, and they observe each other's actions and outcomes, so that they hold a common posterior belief at all times. We let $q_{t}$ denote the agents' belief at time $t$ that the risky arm is good. It follows from Bayes' rule that as long as no Poisson arrival has occurred, this belief evolves according to

$$
d q_{t}=-q_{t}\left(1-q_{t}\right)\left(\sum_{i=1}^{n} a_{i, t}\right) d t
$$

where $a_{i, t}$ denotes agent $i$ 's experimentation level on the risky arm at time $t$. Following a Poisson arrival, the agents learn that the risky arm is good; the (public) belief jumps to 1 and remains there forever. At every moment each agent $i$ observes the common belief $q_{t}$ that the risky arm is good and chooses his experimentation level $a_{i, t}$ at $\operatorname{cost} c_{i}\left(a_{i, t}\right)$, where $c_{i}(\cdot)$ is a strictly increasing, strictly convex, differentiable function satisfying $c_{i}(0)=c_{i}^{\prime}(0)=0$. 
Efficient Mechanism.-First, consider the problem faced by a social planner who chooses the agents' effort levels to maximize social surplus. Her discounted payoff function satisfies the HJB equation

$$
\begin{aligned}
r S(q)= & \max _{a_{1}, . ., a_{n}}\left\{\sum_{i=1}^{n}\left[q a_{i}-c_{i}\left(a_{i}\right)\right]+q\left(\sum_{i=1}^{n} a_{i}\right)[S(1)-S(q)]\right. \\
& \left.-q(1-q)\left(\sum_{i=1}^{n} a_{i}\right) S^{\prime}(q)\right\},
\end{aligned}
$$

where $S(1)=\frac{1}{r} \sum_{i=1}^{n}\left[f_{i}(1)-c_{i}\left(f_{i}(1)\right)\right]$ and $f_{i}(\cdot)=c_{i}^{\prime-1}(\max \{0, \cdot\}) \cdot \cdot^{19}$ The first term on the right side captures the agents' flow payoffs (less their effort cost), the second term captures the expected utility from a Poisson arrival (which will lead the belief to jump from $q$ to 1), and the third term captures the flow cost associated with the agents becoming more pessimistic about the risky arm being good.

We assume that the planner's problem admits a smooth solution and denote each agent's efficient experimentation level by $\bar{a}_{i}(q)$. If the agents have identical experimentation costs, then one can show that in any solution to the planner's problem there exists a threshold $q \in[0,1)$ such that $\bar{a}_{i}(q)>0$ and $\bar{a}_{i}^{\prime}(q)>0$ for all $i$ (and so $S(q)>0)$ if and only if $q>q$. In other words, the agents experiment more intensely the more optimistic they are about the risky arm being good, and below a threshold belief, they stop experimenting altogether. We expect a similar property to hold for the general case with asymmetric agents.

We consider the set of mechanisms that specify flow subsidies $\left\{s_{i}(q)\right\}_{i=1}^{n}$ as a function of the belief $q$ and a prize $V_{i}$ to agent $i$ if he is the first to obtain a Poisson arrival. Given an arbitrary mechanism, agent $i$ 's discounted payoff function satisfies the HJB equation

$$
\begin{aligned}
r J_{i}(q)= & \max _{a_{i}}\left\{q\left(1+V_{i} 1_{\{q<1\}}\right) a_{i}-c_{i}\left(a_{i}\right)\right. \\
& \left.+q\left(\sum_{k=1}^{n} a_{k}\right)\left[J_{i}(1)-J_{i}(q)\right]-q(1-q)\left(\sum_{k=1}^{n} a_{k}\right) J_{i}^{\prime}(q)+s_{i}(q)\right\},
\end{aligned}
$$

where $J_{i}(1)=\frac{1}{r}\left[f_{i}(1)-c_{i}\left(f_{i}(1)\right)\right]$. First, notice from (19) and (20) that following a Poisson arrival (in which case $q=1$ ), the agents exert the efficient experimentation level forever after. Therefore, the subsidies should be equal to 0 whenever $q=1$. Turning to the case in which $q<1$, for the agents to have efficient incentives to experiment, the first-order condition in (20) must match the one in the planner's problem:

$$
S(1)-S(q)-(1-q) S^{\prime}(q)=V_{i}+J_{i}(1)-J_{i}(q)-(1-q) J_{i}^{\prime}(q)
$$

\footnotetext{
${ }^{19}$ If $q=1$, notice that (19) reduces to $r S(1)=\max _{a_{1}, \ldots, a_{n}}\left\{\sum_{i=1}^{n}\left[a_{i}-c_{i}\left(a_{i}\right)\right]\right\}$, so the optimal effort levels satisfy $c_{i}^{\prime}\left(a_{i}\right)=1$, or equivalently, $\bar{a}_{i}(1)=f_{i}(1)$ for all $i$.
} 

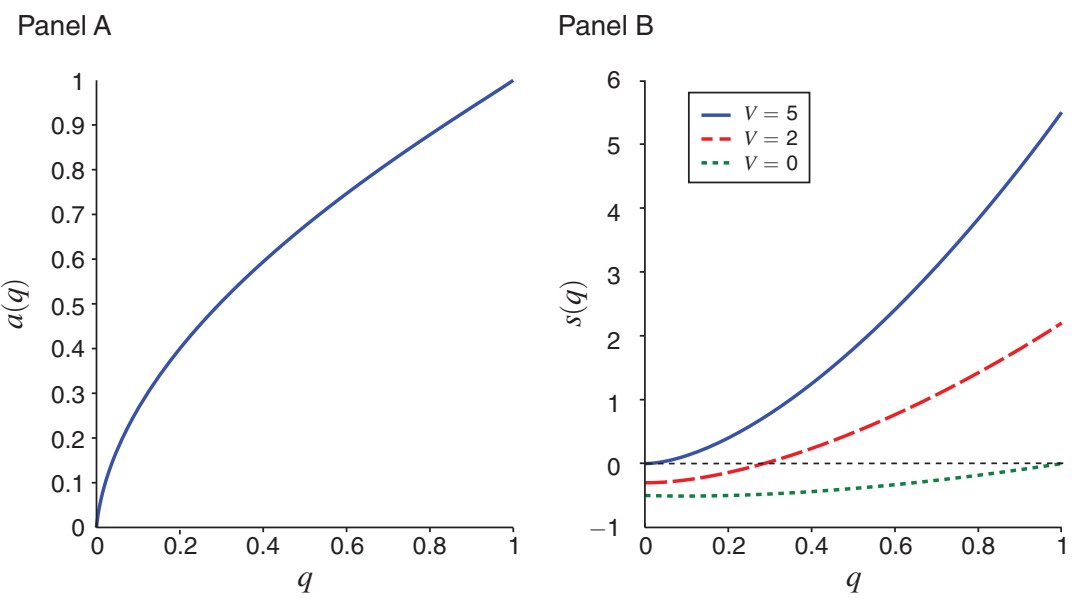

FIGURE 1

Notes: An example of the strategic experimentation problem with $r=0.1, n=5$, and $c_{i}(a)=\frac{a^{2}}{2}$ for all $i$. Panel A illustrates each agent's efficient experimentation level as a function of the public belief $q$. Panel B illustrates the subsidy as a function of the belief $q$ for different choices of $V$.

for all $i$ and $q<1$. This condition is satisfied if $J_{i}(q)=S(q)-\left[S(1)-J_{i}(1)-V_{i}\right]$ for all $i$ and $q$. Solving for $s_{i}(q)$ using (19) and (20) yields

$$
s_{i}(q)=\sum_{k \neq i}\left[q\left(1+V_{i}\right) \bar{a}_{k}(q)-c_{k}\left(\bar{a}_{k}(q)\right)\right]-r\left[S(1)-J_{i}(1)-V_{i}\right]
$$

for all $q<1$, and the first agent who accomplishes a breakthrough receives a lumpsum reward $V_{i}$. Following a breakthrough, it becomes common knowledge that the risky arm is good, each agent exerts the efficient effort level from that time onwards, and no further subsidies are disbursed.

An important assumption is that experimentation levels are observable, which results in the agents holding a common posterior belief at all times. If experimentation levels are not observable as in Bonatti and Hörner (2011), then an agent's present action does not influence the future actions of others. As a result, flow subsidies are unnecessary in this case, and efficiency can be attained either by specifying an appropriate lump-sum reward for the agent who is first to achieve a breakthrough or by using a time-dependent mechanism.

Notice that the efficient mechanism does not pin down the prizes $\left\{V_{i}\right\}_{i=1}^{n}$. As can be seen from $(21)$, the subsidy $s_{i}(\cdot)$ (and hence the implementation cost of the mechanism) increases in the prize $V_{i}$. However, if $V_{i}$ is too small, then the subsidy can become negative (thus amounting to flow payments) for sufficiently pessimistic beliefs. Therefore, the choice of $\left\{V_{i}\right\}_{i=1}^{n}$ must trade off the need to keep the implementation cost of the mechanism low, and to satisfy the agents' cash constraints. Figure 1 uses an example with identical agents and quadratic costs to illustrate how the agents' experimentation levels and the efficiency-inducing subsidies depend on the belief $q$ for different levels of the prize $V$. 
Finally, suppose that budget balance is desired. Then depending on the choice of $\left\{V_{i}\right\}_{i=1}^{n}$, each agent may have to pay an "entry fee" to participate in the efficiency-inducing mechanism, which will be used to fund the subsidies that he will receive in the future. Ex ante budget balance requires that

$$
\sum_{i=1}^{n}\left[J_{i}\left(q_{0}\right)-P_{i, 0}\right]=S\left(q_{0}\right) \Rightarrow \sum_{i=1}^{n}\left(P_{i, 0}+V_{i}\right)=(n-1)\left[S(1)-S\left(q_{0}\right)\right],
$$

and similarly to the previous applications, the individual entry fees and prizes $\left\{P_{i, 0}, V_{i}\right\}_{i=1}^{n}$ will be determined by the way the agents decide to split the profits and the parties' cash constraints.

\section{Discussion}

We study a simple model of dynamic contributions to a public good project. In our model, each of $n$ agents continuously chooses how much effort to allocate to a joint project, and the project generates a lump-sum payoff once the cumulative efforts reach a prespecified threshold. A standard result in such models is that effort is under-provided relative to the efficient outcome due to the free-rider problem. In addition, in our dynamic setting, there is a second form of inefficiency: the agents front-load their effort to induce others to raise their future efforts. We propose a mechanism that induces each agent to always exert the efficient level of effort as the outcome of a Markov perfect equilibrium. The mechanism specifies for each agent flow payments that are due while the project is in progress and a lump-sum reward that is disbursed upon completion of the project.

While we develop our mechanism in the context of a dynamic contributions game, it can be readily applied to other dynamic games with externalities. We illustrate this versatility by adapting it to a dynamic common resource extraction problem, as well as a strategic experimentation problem.

A limitation of our model is that the agents must have sufficient cash in hand at the outset of the game in order to implement the efficient mechanism. In particular, if the project progresses stochastically, then each agent must have unlimited liability. We leave for future work the characterization of the optimal mechanism when each agent has limited cash reserves and the project evolves stochastically. This problem is challenging, because it requires additional state variables: namely, each agent's remaining cash reserves and continuation payoff. This would require extending the approach of Sannikov (2008) to the case of more than one state variable and the case of optimization over both the flow-payments rate and the final payment. Alternatively, the more general Stochastic Maximum Principle (Hamiltonian) approach of Cvitanić and Zhang (2012) might be applied, as in Iijima and Kasahara (2015). 


\section{APPENDIX: PROOFS}

\section{PROOF OF PROPOSITION 1:}

We first study the existence of a solution. We write the ODE system (4) in the form

$$
J_{i}(q)=G_{i}\left(J_{1}^{\prime}(q), \ldots, J_{n}^{\prime}(q)\right)
$$

where $G_{i}\left(J_{1}^{\prime}(q), \ldots, J_{n}^{\prime}(q)\right)=\frac{1}{r}\left\{-c_{i}\left(f_{i}\left(J_{i}^{\prime}\right)\right)+\left[\sum_{j=1}^{n} f_{j}\left(J_{j}^{\prime}\right)\right] J_{i}^{\prime}\right\}$. Since the FOC is $c_{i}^{\prime}\left(a_{i}(q)\right)=J_{i}^{\prime}(q)$ and $c_{i}^{\prime}>0$, we are looking for a solution such that $\min _{j} J_{j}^{\prime}>0$. Thus, we consider $G_{i}$ only on the domain $+n=(0, \infty)^{n}$. It follows then from (4) and the monotonicity of $x f_{i}(x)-c_{i}\left(f_{i}(x)\right)$ that $\min _{j} J_{j}>0$.

We need the following lemma.

LEMMA 3: The mapping $G=\left(G_{1}, \ldots, G_{n}\right)$ from $+n$ to $+n$ is invertible and the inverse mapping $F$ is continuously differentiable. Moreover, $\frac{\partial F_{i}}{\partial x_{i}}>0$, that is, the $i$-th component of the inverse mapping is strictly increasing in the $i$-th variable.

\section{PROOF OF LEMMA 3:}

By the Gale-Nikaido generalization of Inverse Function Theorem (see, for example, theorem 20.4 in Nikaido 1968), for the first part of the lemma it is sufficient to show that all the principal minors of the Jacobian matrix of the mapping $G$ are strictly positive for all $\left(J_{1}^{\prime}, \ldots, J_{n}^{\prime}\right) \in+n$. We sketch the proof. The $1 \times 1$ principal minors, that is, the diagonal entries $\frac{1}{r} \sum_{j=1}^{n} f_{j}\left(J_{j}^{\prime}\right)$ of the Jacobian are positive (for any $n$ ). For $n=2$, from the expressions for the partial derivatives of $G_{i}$, we see that the determinant of the Jacobian is proportional to

$$
\left(\sum_{j=1}^{2} f_{j}\left(J_{j}^{\prime}\right)\right)^{2}-f_{1}^{\prime}\left(J_{1}^{\prime}\right) f_{2}^{\prime}\left(J_{2}^{\prime}\right) J_{1}^{\prime} J_{2}^{\prime}>0
$$

where the inequality holds on the domain +2 , by noting that $f_{i}(x)>x f_{i}^{\prime}(x)$ for all $i$ and $x>0 .{ }^{20}$ Straightforward, but tedious computations show that the determinants for $n=3$ and $n=4$ are proportional to

$$
\sum_{j=1}^{3} f_{j}\left(J_{j}^{\prime}\right)\left[\left(\sum_{j=1}^{3} f_{j}\left(J_{j}^{\prime}\right)\right)^{2}-\sum_{i<j} f_{i}^{\prime}\left(J_{i}^{\prime}\right) f_{j}^{\prime}\left(J_{j}^{\prime}\right) J_{i}^{\prime} J_{j}^{\prime}\right]+2 \Pi_{k=1}^{3} f_{k}^{\prime}\left(J_{k}^{\prime}\right) J_{k}^{\prime}
$$

${ }^{20}$ To see why, first note that for all $x>0$ we have $f_{i}^{\prime}\left(c_{i}^{\prime}(x)\right)=\frac{1}{c_{i}^{\prime \prime}(x)}$ and so $f_{i}^{\prime \prime}\left(c_{i}^{\prime}(x)\right)=-\frac{c_{i}^{\prime \prime \prime}(x)}{\left[c_{i}^{\prime \prime}(x)\right]^{2}} \leq 0$. Next, let $k_{i}(x)=f_{i}(x)-x f_{i}^{\prime}(x)$, and observe that $k_{i}(0)=0$ by assumption and $k_{i}^{\prime}(x)=-x f_{i}^{\prime \prime}(x) \geq 0$ for all $x>0$. Therefore, $f_{i}(x)>x f_{i}^{\prime}(x)$ for all $x>0$. 
and, respectively,

$$
\begin{aligned}
& \left(\sum_{j} f_{j}\left(J_{j}^{\prime}\right)\right)^{2}\left[\left(\sum_{j} f_{j}\left(J_{j}^{\prime}\right)\right)^{2}-\sum_{i<j} f_{i}^{\prime} f_{j}^{\prime} J_{i}^{\prime} J_{j}^{\prime}\right]+2 \sum_{i<j<k} f_{i}^{\prime} f_{j}^{\prime} f_{k}^{\prime} J_{i}^{\prime} J_{j}^{\prime} J_{k}^{\prime} \sum_{j} f_{j}\left(J_{j}^{\prime}\right) \\
- & 3 f_{1}^{\prime} f_{2}^{\prime} f_{3}^{\prime} f_{4}^{\prime} J_{1}^{\prime} J_{2}^{\prime} J_{3}^{\prime} J_{4}^{\prime}
\end{aligned}
$$

Both of these expressions are positive, by a similar argument as for $n=2$. In general, it can be verified that the determinant of the $n$-dimensional Jacobian has the term

$$
\left(\sum_{j} f_{j}\left(J_{j}^{\prime}\right)\right)^{n-2}\left[\left(\sum_{j} f_{j}\left(J_{j}^{\prime}\right)\right)^{2}-\sum_{i<j} f_{i}^{\prime} f_{j}^{\prime} J_{i}^{\prime} J_{j}^{\prime}\right],
$$

which is positive, and that the remaining terms are of the form, for $k=3,4, \ldots, n$,

$$
C_{k} \sum_{i_{1}<\ldots<i_{k}} f_{i_{1}}^{\prime} J_{i_{1}}^{\prime} \cdots f_{i_{k}}^{\prime} J_{i_{k}}^{\prime}\left(\sum_{j} f_{j}\left(J_{j}^{\prime}\right)\right)^{n-k},
$$

where $C_{k}>0$ for $k$ odd, and $C_{k}<0$ for $k$ even, and the values of $C_{k}$ are such that the positive terms dominate the negative terms (when we take into account that $\left.f_{i}(x)>x f_{i}^{\prime}(x)\right)$.

Similar computations show that not only the determinants, but all the principal minors are positive.

For the last statement of the lemma, by the Inverse Function Theorem, we need to show that the diagonal entries of the inverse of the Jacobian matrix are strictly positive. By Cramer's rule, those entries are proportional to the diagonal entries of the adjugate of the Jacobian, thus proportional to the diagonal entries of the cofactor matrix of the Jacobian. Those entries are equal to the corresponding $(n-1)$ $\times(n-1)$ principal minors, which, by the argument above, are positive.

This lemma asserts that, if the values $J_{i}$ and the marginal values $J_{i}^{\prime}$ solve the HJB equation, then they are in one-to-one smooth strictly increasing correspondence, and so are the optimal actions $a_{i}$. That is, increasing an agent's action also increases his value function, and vice versa (holding everything else constant).

Next, we show that the set of solutions to the system of ODE's is not empty.

LEMMA 4: For every $\epsilon \in\left(0, \min _{i}\left\{V_{i}\right\}\right)$, there exists some $Q_{\epsilon}>0$ such that there exists a unique solution $\left(J_{1}, \ldots, J_{n}\right)$ to the ODE system on interval $\left[0, Q_{\epsilon}\right]$ that satisfies $J_{i} \geq \epsilon$ on that interval, for all $i$.

PROOF OF LEMMA 4:

The ODE system (4) can be written as

$$
J_{i}^{\prime}(q)=F_{i}\left(J_{1}(q), \ldots, J_{n}(q)\right)
$$


For given $\epsilon>0$, denote

$$
M_{F}=\max _{i} \max _{\epsilon \leq x_{i} \leq V_{i}} F_{i}\left(x_{1}, \ldots, x_{n}\right) .
$$

Pick $Q_{\epsilon}$ sufficiently close to zero so that, for all $i$,

$$
V_{i}-\left(Q_{\epsilon}\right) M_{F} \geq \epsilon
$$

Then, define $\Delta q=\frac{Q_{\epsilon}}{N}$ and functions $J_{i}^{N}$ by Picard iterations, going backwards from $Q_{\epsilon}$,

$$
\begin{aligned}
J_{i}^{N}\left(Q_{\epsilon}\right) & =V_{i} \\
J_{i}^{N}\left(Q_{\epsilon}-\Delta q\right) & =V_{i}-\Delta q F_{i}\left(V_{1}, \ldots, V_{n}\right) \\
J_{i}^{N}\left(Q_{\epsilon}-2 \Delta q\right) & =J_{i}^{N}\left(Q_{\epsilon}-\Delta q\right)-\Delta q F_{i}\left(J_{1}^{N}\left(Q_{\epsilon}-\Delta q\right), \ldots, J_{n}^{N}\left(Q_{\epsilon}-\Delta q\right)\right) \\
& =V_{i}-\Delta q F_{i}\left(V_{1}, \ldots, V_{n}\right)-\Delta q F_{i}\left(J_{1}^{N}\left(Q_{\epsilon}-\Delta q\right), \ldots, J_{n}^{N}\left(Q_{\epsilon}-\Delta q\right)\right)
\end{aligned}
$$

and so on, until $J_{i}^{N}\left(Q_{\epsilon}-N \Delta q\right)=J_{i}(0)$. Then, we complete the definition of function $J_{i}^{N}$ by making it piecewise linear between the points $Q_{\epsilon}-k \Delta q$, $k=1, \ldots, N$. Notice, from the assumption on $Q_{\epsilon}$ that $J_{i}^{N}(V-k \Delta q) \geq \epsilon$, for all $k=1, \ldots, N$. Since $F_{i}$ are continuously differentiable, they are Lipschitz on the $n$-dimensional bounded domain $\Pi_{j=1}^{n}\left[\epsilon, V_{i}\right]$. Thus, by the standard ODE argument, $\left\{J_{i}^{N}\right\}$ converge to a unique solution $\left\{J_{i}\right\}$ of the ODE system on $q \in\left[0, Q_{\epsilon}\right]$, and we have $J_{i} \geq \epsilon$.

Lemma 4 shows that the system of ODE's has a unique solution on $\left[0, \sup _{\epsilon} Q_{\epsilon}\right)$.

Next, fixing $Q<\sup _{\epsilon} Q_{\epsilon}$, we now want to show that the ODE solution $J_{i}(q)$ is the value function of agent $i$, for every initial project value $q_{0} \in[0, Q]$, if every other agent plays $a_{j}(q)=J_{j}^{\prime}(q)$. We have, for any action $a_{i}\left(q_{t}\right)$,

$$
\begin{aligned}
e^{-r \tau} J_{i}(Q) & =J_{i}\left(q_{0}\right)+\int_{0}^{\tau} d\left(e^{-r t} J_{i}\left(q_{t}\right)\right) \\
& =J_{i}\left(q_{0}\right)+\int_{0}^{\tau} e^{-r t}\left[-r J_{i}\left(q_{t}\right)+J_{i}^{\prime}\left(q_{t}\right) \sum_{j=1}^{n} a_{j}\left(q_{t}\right)\right] d t \\
& \leq J_{i}\left(q_{0}\right)+\int_{0}^{\tau} e^{-r t} c_{i}\left(a_{i}\left(q_{t}\right)\right) d t
\end{aligned}
$$

where the last inequality is due to the HJB equation (2). Since $J_{i}(Q)=\alpha_{i} V$, this implies that the agent's value function, denoted $J_{i}^{*}$, satisfies $J_{i}^{*}\left(q_{0}\right) \leq J_{i}\left(q_{0}\right)$. Moreover, the upper bound is attained if the agent plays $a_{i}(q)=J_{i}^{\prime}(q)$. 
Note that since the inverse $F$ of $G$ is differentiable, so are the functions $J_{i}^{\prime}$. To establish convexity, we differentiate the ODE for $J_{i}$ to obtain

$$
r J_{i}^{\prime}=-J_{i}^{\prime} f_{i}^{\prime} J_{i}^{\prime \prime}+J_{i}^{\prime} \sum_{j=1}^{n} f_{j}^{\prime}\left(J_{j}^{\prime}\right) J_{j}^{\prime \prime}+J_{i}^{\prime \prime} \sum_{j=1}^{n} f_{j}\left(J_{j}^{\prime}\right) .
$$

We can view this as a linear system for the vector $J^{\prime \prime}$ with entries $J_{i}^{\prime \prime}$, which can be written as $M J^{\prime \prime}=r J^{\prime}$, where $J^{\prime}$ denotes the vector with entries $J_{i}^{\prime}$, and the $i$-th row of matrix $M$ is

$$
\left(J_{i}^{\prime} f_{1}\left(J_{1}^{\prime}\right), . ., J_{i}^{\prime} f_{i-1}\left(J_{i-1}^{\prime}\right), \sum_{j=1}^{n} f_{j}\left(J_{j}^{\prime}\right), J_{i}^{\prime} f_{i+1}\left(J_{i+1}^{\prime}\right), . ., J_{i}^{\prime} f_{n}\left(J_{n}^{\prime}\right)\right)
$$

Using the main result of Kaykobad (1985), a sufficient condition for $J^{\prime \prime}$ to have all strictly positive entries is that $J^{\prime}$ has all strictly positive entries and

$$
\sum_{j=1}^{n} f_{j}\left(J_{j}^{\prime}\right)>\sum_{j \neq i} J_{j}^{\prime} f_{j}^{\prime}\left(J_{j}^{\prime}\right)
$$

which holds if $f_{j}(x) \geq x f_{j}^{\prime}(x)$ for all $x \geq 0$ and $j$. By noting that this condition is always satisfied (see footnote 20 for details), the proof is complete.

\section{PROOF OF PROPOSITION 2:}

To study the existence of a solution, we proceed as in Proposition 1. First, we write the system of ODE's (7) in the form

$$
r \bar{S}(q)=G\left(\bar{S}^{\prime}(q)\right)
$$

for an appropriate function $G$. As in the MPE case, we can show that $G(x)$ has a continuously differentiable and strictly increasing inverse function $F(y)$, for $y>0$. Thus, $\bar{S}$ is twice differentiable and convex, and the rest of the proof is similar to the proof of Proposition 1, and we omit the details.

\section{PROOF OF REMARK 1:}

Let $\bar{S}_{n}(q)$ denote the social planner's value function when the group comprises of $n$ symmetric agents, and observe that $\bar{S}_{n}(q)$ is nondecreasing in $n$. That is because the social planner can always instruct the additional agent(s) to exert zero effort without decreasing her payoff. Then, it follows from $(7)$ that $G\left(\bar{S}_{n}^{\prime}(q)\right)$ also increases in $n$, where

$$
G(x)=n[f(x) x-c(f(x))]
$$

Now consider the change of variables

$$
y=n f(x) \text { so that } x=c^{\prime}\left(\frac{y}{n}\right),
$$


and the function

$$
\tilde{G}(y)=y c^{\prime}\left(\frac{y}{n}\right)-n c\left(\frac{y}{n}\right)
$$

Observe that $\tilde{G}^{\prime}(y)=c^{\prime}\left(\frac{y}{n}\right)+\frac{y}{n} c^{\prime \prime}\left(\frac{y}{n}\right)-c^{\prime}\left(\frac{y}{n}\right)=\frac{y}{n} c^{\prime \prime}\left(\frac{y}{n}\right)>0$, so $\tilde{G}(y)$ is increasing in $y=n f(x)$. To show that $n\left(f\left(\bar{S}_{n}^{\prime}(q)\right)\right)$ increases in $n$ for all $q$, suppose that the contrary is true, i.e., that there exists some $q$ for which $n\left(f\left(\bar{S}_{n}^{\prime}(q)\right)\right)$ decreases in $n$. Then $\tilde{G}\left(n\left(f\left(\bar{S}_{n}^{\prime}(q)\right)\right)\right)$ must also decrease in $n$. However, this is a contradiction, because $\tilde{G}\left(n\left(f\left(\bar{S}_{n}^{\prime}(q)\right)\right)\right)=\bar{S}_{n}(q)$. Therefore, $q_{t}=\int_{0}^{t} n\left(f\left(\bar{S}_{n}^{\prime}\left(q_{s}\right)\right)\right) d s$ also increases in $n$, which in turn implies that the completion time $\bar{\tau}$ decreases in $n$.

\section{PROOF OF PROPOSITION 3:}

Fix an arbitrary $q$ and $i$. Note that the function

$$
G_{i}(x)=x f_{i}(x)-c_{i}\left(f_{i}(x)\right)
$$

is strictly increasing in $x>0$. To show that the efficient effort is higher than the minimal MPE effort, let $k$ be the index $j$ for which $J_{j}^{\prime}(q)$ is the lowest. We have, from HJB equations (4) and (7),

$$
\begin{aligned}
r\left[\bar{S}(q)-J_{k}(q)\right] \leq & \sum_{j \neq k}\left[f_{j}\left(\bar{S}^{\prime}(q)\right) \bar{S}^{\prime}(q)-f_{j}\left(J_{k}^{\prime}(q)\right) J_{k}^{\prime}(q)-c_{j}\left(f_{j}\left(\bar{S}^{\prime}(q)\right)\right)\right] \\
& +G_{k}\left(\bar{S}^{\prime}(q)\right)-G_{k}\left(J_{k}^{\prime}(q)\right) .
\end{aligned}
$$

Thus, if $\bar{S}^{\prime}(q)<J_{k}^{\prime}(q)$, then we would also have $\bar{S}(q)<J_{k}(q)$, which is impossible, because $\bar{S}(q)$ is the optimal social welfare. Therefore, $\bar{S}^{\prime}(q) \geq J_{k}^{\prime}$ $(q)$, and hence $\bar{a}_{k}(q) \geq a_{k}(q)$ for all $q$. Therefore, if the agents are symmetric (i.e., $\alpha_{i}=\alpha_{j}$ and $c_{i}(a)=c_{j}(a)$ for all $i, j$, and $\left.a\right)$, then $\bar{S}^{\prime}(q) \geq J_{i}^{\prime}(q)$, and hence $\bar{a}_{i}(q)$ $\geq a_{i}(q)$ for all $i$ and $q$.

\section{PROOF OF PROPOSITION 4:}

Since we have

$$
c_{i}^{\prime}\left(f_{i}\left(J_{i}^{\prime}(q)\right)=J_{i}^{\prime}(q) \text { and } q^{\prime}=\sum_{j} f_{j}(q)\right.
$$

we get

$$
\frac{d}{d t} e^{-r t} c_{i}^{\prime}\left(f_{i}\left(J_{i}^{\prime}\left(q_{t}\right)\right)=e^{-r t}\left[-r J_{i}^{\prime}\left(q_{t}\right)+J_{i}^{\prime \prime}(q) \sum_{j} f_{j}\left(q_{t}\right)\right] .\right.
$$

On the other hand, differentiating the ODE for $J_{i}$ we obtain

$$
r J_{i}^{\prime}=J_{i}^{\prime} \sum_{j \neq i}^{n} f_{j}^{\prime}\left(J_{j}^{\prime}\right) J_{j}^{\prime \prime}+J_{i}^{\prime \prime} \sum_{j=1}^{n} f_{j}\left(J_{j}^{\prime}\right) .
$$


Thus, we have

$$
\frac{d}{d t} e^{-r t} c_{i}^{\prime}\left(f_{i}\left(J_{i}^{\prime}\left(q_{t}\right)\right)=-e^{-r t} J_{i}^{\prime}\left(q_{t}\right) \sum_{j \neq i}^{n} f_{j}^{\prime}\left(J_{j}^{\prime}\left(q_{t}\right) J_{j}^{\prime \prime}\left(q_{t}\right)\right.\right.
$$

From Proposition 1, we know that this is strictly negative. Similarly, differentiating the ODE for the social planner's value function $\bar{S}$, we get

$$
r \bar{S}^{\prime}=\bar{S}^{\prime \prime} \sum_{j=1}^{n} f_{j}\left(\bar{S}^{\prime}\right)
$$

which implies

$$
\frac{d}{d t} e^{-r t} c_{i}^{\prime}\left(f_{i}\left(\bar{S}^{\prime}\left(q_{t}\right)\right)\right)=0
$$

PROOF OF LEMMA 1:

To establish existence of an MPE that implements the efficient outcome, substitute (9) into (8) and take agent $i$ 's first-order condition. This yields the system of ODE

$$
\begin{aligned}
r \hat{J}_{i}(q) & =-c_{i}\left(f_{i}\left(\hat{J}_{i}^{\prime}(q)\right)\right)+\left[\sum_{j=1}^{n} f_{j}\left(\hat{J}_{i}^{\prime}(q)\right)\right] \hat{J}_{i}^{\prime}(q)-\sum_{j \neq i} c_{j}\left(f_{j}\left(\bar{S}^{\prime}(q)\right)\right) \\
& =\left[\sum_{j=1}^{n} f_{j}\left(\hat{J}_{i}^{\prime}(q)\right)\right] \hat{J}_{i}^{\prime}(q)-\sum_{j=1}^{n} c_{j}\left(f_{j}\left(\bar{S}^{\prime}(q)\right)\right)
\end{aligned}
$$

subject to $\hat{J}_{i}(Q)=V$. It follows from (7) that $\hat{J}_{i}(q)=S(q)$ satisfies this ODE for all $i$ and $q \in[0, Q]$. Because the planner's problem has a unique solution (by Proposition 2), it follows that this is the unique project-completing MPE.

Part (ii) of Lemma 1 follows by noting that $f_{i}(\cdot)$ is increasing for all $i, \bar{S}^{\prime}(q)>0$ and $\bar{S}^{\prime \prime}(q)>0$ for all $q>\underline{q}^{s}$, and so that $h_{i}^{\prime}(q)=\bar{S}^{\prime}(q) \bar{S}^{\prime \prime}(q) \sum_{j \neq i} f_{j}^{\prime}\left(\bar{S}^{\prime}(q)\right)>0$.

\section{PROOF OF LEMMA 2:}

Noting that the balance in the savings account evolves according to $d H_{t}$ $=\left[r H_{t}+\sum_{j=1}^{n} h_{j}\left(q_{t}\right)\right] d t$, each agent $i$ 's problem is

$$
r J_{i}(q, H)=\max _{a_{i}}\left\{-c_{i}\left(a_{i}\right)+\left(\sum_{j=1}^{n} a_{j}\right) \frac{J_{i}(q, H)}{\partial q}+\left(r H+\sum_{j=1}^{n} h_{j}\right) \frac{J_{i}(q, H)}{\partial H}-h_{i}(q)\right\}
$$

subject to the boundary condition

$$
J_{i}(Q, H)=\beta_{i}(V+H)
$$


Note that here, each agent's discounted payoff function $J_{i}$ is a function of both the state of the project $q$ and the balance in the savings account $H$. We conjecture that $J_{i}$ is of the form

$$
J_{i}(q, H)=\beta_{i} H+\tilde{J}_{i}(q)
$$

in which case the HJB equation for $\tilde{J}_{i}(q)$ can be rewritten as

$$
r \tilde{J}_{i}(q)=\max _{a_{i}}\left\{-c_{i}\left(a_{i}\right)+\left(\sum_{j=1}^{n} a_{j}\right) \tilde{J}_{i}^{\prime}(q)-\left[h_{i}(q)-\beta_{i} \sum_{j=1}^{n} h_{j}(q)\right]\right\}
$$

subject to

$$
\tilde{J}_{i}(Q)=\beta_{i} V
$$

The first-order condition gives $a_{i}(q)=f_{i}\left(\tilde{J}_{i}^{\prime}(q)\right)$ so that

$$
r \tilde{J}_{i}(q)=-c_{i}\left(f_{i}\left(\tilde{J}_{i}^{\prime}(q)\right)\right)+\left[\sum_{j=1}^{n} f_{j}\left(\tilde{J}_{j}^{\prime}(q)\right)\right] \tilde{J}_{i}^{\prime}(q)-\left[h_{i}(q)-\beta_{i} \sum_{j=1}^{n} h_{j}(q)\right] .
$$

As before, this ODE system has a unique solution, and it is straightforward to verify that $\beta_{i} H+\tilde{J}_{i}$ solves the HJB equation for $J_{i}(q, H)$. Hence, the conjecture has been justified. Notice that from $(23)$ that $\tilde{J}_{i}(\cdot)$ corresponds to the value function of agent $i$ who receives $\beta_{i} V$ upon completion of the project and makes flow payments $\tilde{h}_{i}(q)$ $=h_{i}(q)-\beta_{i} \sum_{j=1}^{n} h_{j}(q)$. These flow payments add up to zero, and the proposition is proved.

\section{PROOF OF PROPOSITION 5:}

Let $\bar{J}_{j}(q)$ denote agent $j$ 's discounted payoff function for the problem in which each agent $j$ plays the efficient strategy $\bar{a}_{j}(q)$, he makes flow payments $h_{j}(q)=\sum_{j \neq i} c_{j}\left(f_{j}\left(\bar{S}^{\prime}(q)\right)\right)+r p_{j}$, and receives $V-p_{j}$ upon completion, where $\sum_{j=1}^{n} p_{j}=(n-1) \bar{S}(0)$. Fix an agent $i$, and assume that every agent $j \neq i$ plays $\bar{a}_{j}(q)=f_{j}\left(S^{\prime}(q)\right)$. Moreover, let $\beta_{i}=\frac{V-p_{i}}{V+(n-1)[V-\bar{S}(0)]}$. We have, for any effort $a_{i}(q)$ process of agent $i$, with $\tau^{a}$ denoting the corresponding completion time,

$$
\begin{aligned}
& e^{-r \tau^{a}} \min \left\{V-p_{i}, \beta_{i}\left(V+H_{\tau}\right)\right\}-\int_{u}^{\tau^{a}} e^{-r s}\left\{h_{i}\left(q_{s}\right)+c_{i}\left(a_{i}\left(q_{s}\right)\right)\right\} d s \\
\leq & e^{-r \tau^{a}}\left(V-p_{i}\right)-\int_{u}^{\tau^{a}} e^{-r s}\left\{h_{i}\left(q_{s}\right)+c_{i}\left(a_{i}\left(q_{s}\right)\right)\right\} d s \\
\leq & e^{-r u} \bar{J}_{i}\left(q_{u}\right),
\end{aligned}
$$

where the last inequality holds because $\bar{J}_{i}$ is the value function of the problem with the final payment $\bar{J}_{i}(\tau)=V-p_{i}$. In particular, with $u=t$, we see 
that $\bar{J}_{i}\left(q_{t}\right)$ is an upper bound also for agent $i$ 's problem with the final payment $\min \left\{V-p_{i}, \beta_{i}\left(V+H_{\tau}\right)\right\}$. On the other hand, with $a_{i}(q)=\bar{a}_{i}(q)$ for all $q$, all the inequalities above become equalities, so it is optimal for agent $i$ to choose strategy $\bar{a}_{i}(q)$ also in the game with the final payment $\min \left\{V-p_{i}, \beta_{i}\left(V+H_{\tau}\right)\right\}$. We see that this is also true starting from any point in time $t \leq u \leq \tau$, thus all playing $\bar{a}_{i}(q)$ is an MPE. In the above argument we implicitly assumed that $\tau^{a}$ is finite. If $\tau^{a}$ is infinite, agent $i$ 's value function is negative, thus inferior to $\bar{J}(q)$.

Since each agent's reward is capped by $\beta_{i}\left(V+H_{\tau}\right)$, the mechanism will never result in a budget deficit. Moreover, since on the equilibrium path each agent $i$ collects $V-p_{i}$ upon completion, and the $p_{i}$ s are chosen so that the the budget is balanced, part (ii) follows. Part (iii) follows directly from the construction in Section IIIA.

\section{PROOF OF PROPOSITION 7:}

Suppose first that each agent $i$ receives zero if he runs out of cash before the project is completed, and otherwise he gets exactly $V$ upon completion. Then, the ODE system looks the same as before, except $h_{i}(q)$ in the ODE is multiplied by $I_{i}(q)$ and $V$ in the terminal condition is multiplied by $I_{i}(Q)$. The efficient outcome is still an MPE, because with the corresponding choice of effort, $I_{i}(q)=1$ for all $i$ and all $q \leq Q$, and the system of ODE is the same as before.

Suppose now that each agent $i$ is promised $\min \left\{V, \frac{V+H_{\tau}}{n}\right\}$ instead of $V$ at completion, and zero if he runs out of cash. Then, the agent's payoff is equal to

$$
\begin{aligned}
& I_{i}(Q) e^{-r \tau} \min \left\{V-p_{i}, \beta_{i}\left(V+H_{\tau}\right)\right\}-\int_{0}^{\tau} e^{-r s}\left\{I_{i}\left(q_{s}\right) h_{i}\left(q_{s}\right)+c_{i}\left(a_{i}\left(q_{s}\right)\right)\right\} d s \\
& \quad \leq I_{i}(Q) e^{-r \tau}\left(V-p_{i}\right)-\int_{0}^{\tau} e^{-r s}\left\{I_{i}\left(q_{s}\right) h_{i}\left(q_{s}\right)+c_{i}\left(a_{i}\left(q_{s}\right)\right)\right\} d s .
\end{aligned}
$$

Consider first the deviations in which the agent does not run out of cash by completion. Then, as before, with $\bar{J}_{i}(q)$ denoting agent $i$ 's value function in the efficient MPE, the last term is no greater than $\bar{J}_{i}(0)$, and hence the agent would not deviate if no one else does. If the agent applies a deviation in which he runs out of cash, then his value function is negative, and the same conclusion holds.

\section{PROOF OF COROLLARY 1:}

We first show that $\hat{g}_{i}(\tau)$ is nonincreasing in $\tau$. For all $\tau$ such that $g_{i}(\tau) \leq$ $\alpha_{i} V \leq V$, because $\sum_{j \neq i} c_{j}\left(f_{j}\left(\bar{S}^{\prime}(Q)\right)\right)>0$ for all $i$, it follows from (14) that $g_{i}^{\prime}(\tau)<0$, and so $\hat{g}_{i}^{\prime}(\tau) \leq 0$. On the other hand, for $\tau$ such that $g_{i}(\tau)>\alpha_{i} V$, we have $\hat{g}_{i}(\tau)=\alpha_{i} V$ and so it is independent of $\tau$. From this it follows that for $\tau<\bar{\tau}$, we have $g_{i}(\tau)>\alpha_{i} V$. Because, if we had $g_{i}(\tau) \leq \alpha_{i} V$ for some $\tau<\bar{\tau}$, then, $g_{i}(\cdot)$ would be strictly decreasing on $s>\tau$, and we could not have $g_{i}(\bar{\tau})=\alpha_{i} V$.

Next, let $\bar{J}_{i}(t, q)$ denote agent $i$ 's discounted payoff function corresponding to the game in which each agent $i$ pays $h_{i}(t, q)$ and receives $g_{i}(\tau)$ at completion time $\tau$, 
and agents $j \neq i$ play the first-best strategies. Then, by the arguments in Section IV, agent $i$ also plays first-best strategy $a_{i}(t, q)=\bar{S}^{\prime}(q)$.

We aim to show that if each agent makes flow payments $\hat{h}_{i}(t, q)=\left[h_{i}(t, q)\right]^{+}$and receives $\hat{g}_{i}(\tau)=\left[\min \left\{\alpha_{i} V, g_{i}(\tau)\right\}\right]^{+}$upon completion of the project, and all agents except for $i$ play first-best strategies, then agent $i$ cannot do better than playing first best himself. There are three cases to consider:

(i) If the effort process $a_{i}(t, q)$ of agent $i$ does not lead to completion, then he has to pay nonnegative payments forever but will never receive a lump-sum reward, which is worse than playing first best.

(ii) Similarly, playing $a_{i}(t, q)$ such that the corresponding completion time $\tau^{a}$ is larger than or equal to $\tau_{i}^{0}$, where $\tau_{i}^{0}=\min \left\{\tau: g_{i}(\tau)=0\right\}$, is worse than first best, because he gets zero reward upon completion.

(iii) Suppose now he plays $a_{i}(t, q)$ such that the corresponding completion time $\tau^{a}$ is less than $\tau_{i}^{0}$. Then, for $\tau<\tau_{i}^{0}$, we have $\hat{g}_{i}(\tau)=\min \left\{\alpha_{i} V, g_{i}(\tau)\right\}$ and the discounted payoff satisfies

$$
\begin{aligned}
& e^{-r \tau^{a}} \hat{g}_{i}\left(\tau^{a}\right)-\int_{t}^{\tau^{a}} e^{-r s}\left\{h^{+}\left(s, q_{s}\right)+c_{i}\left(a_{i}\left(s, q_{s}\right)\right)\right\} d s \\
& \quad \leq e^{-r \tau^{a}} g_{i}\left(\tau^{a}\right)-\int_{t}^{\tau^{a}} e^{-r s}\left\{h_{i}\left(s, q_{s}\right)+c_{i}\left(a_{i}\left(q_{s}\right)\right)\right\} d s \\
& \quad \leq e^{-r t} \bar{J}_{i}\left(t, q_{t}\right),
\end{aligned}
$$

where the last equality holds because $\bar{J}_{i}$ is the value function of the problem of maximizing the expression in the second line. In particular, with $t=0$, we see that $\bar{J}_{i}(0,0)$ is an upper bound for agent $i$ 's payoff. Moreover, with all other agents playing the first-best strategy, if agent $i$ also plays first best, we have $\tau^{a}=\bar{\tau}, g_{i}(\bar{\tau})$ $=\hat{g}_{i}(\bar{\tau})=\alpha_{i} V, h_{i} \equiv \hat{h}_{i} \equiv 0$, and all the inequalities above become equalities, so it is optimal for agent $i$ to also choose first-best strategy $\bar{S}^{\prime}(q)$.

Altogether, we see that under the mechanism $\{\hat{g}, \hat{h}\}$ playing first best is an MPE, in which case the budget is balanced on the equilibrium path, and there is no budget deficit even off equilibrium path.

\section{REFERENCES}

Admati, Anat R., and Motty Perry. 1991. "Joint Projects without Commitment." Review of Economic Studies 58 (2): 259-76.

Alchian, Armen A., and Harold Demsetz. 1972. "Production, Information Costs, and Economic Organization." American Economic Review 62 (5): 777-95.

Athey, Susan, and Ilya Segal. 2007. “An Efficient Dynamic Mechanism.” Paper presented at Choice, Contracts and Computation 2008, Yale University, New Haven, CT, June 9-10.

Athey, Susan, and Ilya Segal. 2013. “An Efficient Dynamic Mechanism.” Econometrica 81 (6): $2463-85$.

-Bagnoli, Mark, and Barton L. Lipman. 1989. "Provision of Public Goods: Fully Implementing the Core through Private Contributions.” Review of Economic Studies 56 (4): 583-601. 
Bergemann, Dirk, and Ulrich Hege. 2005. "The Financing of Innovation: Learning and Stopping." RAND Journal of Economics 36 (4): 719-52.

-Bergemann, Dirk, and Juuso Välimäki. 2010. "The Dynamic Pivot Mechanism.” Econometrica 78 (2): 771-89.

-Bonatti, Alessandro, and Johannes Hörner. 2011. "Collaborating." American Economic Review 101 (2): 632-63.

Cvitanić, Jakša, and Jianfeng Zhang. 2012. Contract Theory in Continuous-Time Models. New York: Springer Finance.

Georgiadis, George. 2015a. "Deadlines and Infrequent Monitoring in the Dynamic Provision of Public Goods." http://papers.ssrn.com/sol3/papers.cfm?abstract_id=2618822.

-Georgiadis, George. 2015b. "Projects and Team Dynamics." Review of Economic Studies 82 (1): $187-218$

Georgiadis, George, Steven A. Lippman, and Christopher S. Tang. 2014. "Project design with limited commitment and teams." RAND Journal of Economics 45 (3): 598-623.

Halac, Marina, Navin Kartik, and Qingmin Liu. 2015. "Optimal Contracts for Experimentation." http://www.columbia.edu/ nk2339/Papers/HKL_contracts_experimentation.pdf.

Harvard Business Review. 2004. "Managing Teams: Forming a Team that Makes a Difference." In Manager's Toolkit: The 13 Skills Managers Need to Succeed, 93-114. Boston: Harvard Business School Press.

-Holmström, Bengt. 1982. "Moral Hazard in Teams.” Bell Journal of Economics 13 (2): 324-40.

- Ichniowski, Casey, and Kathryn Shaw. 2003. "Beyond Incentive Pay: Insiders' Estimates of the Value of Complementary Human Resource Management Practices." Journal of Economic Perspectives 17 (1): 155-80.

Iijima, Ryota, and Akitada Kasahara. 2015. "Gradual Adjustment and Equilibrium Uniqueness under Noisy Monitoring." http://scholar.harvard.edu/files/riijima/files/jmp1215.pdf.

-Kaykobad, M. 1985. "Positive Solutions of Positive Linear Systems." Linear Algebra and its Applications 64: 133-40.

Keller, Godfrey, Sven Rady, and Martin Cripps. 2005. "Strategic Experimentation with Exponential Bandits." Econometrica 73 (1): 39-68.

- Kessing, Sebastian G. 2007. "Strategic Complementarity in the Dynamic Private Provision of a Discrete Public Good." Journal of Public Economic Theory 9 (4): 699-710.

-Legros, Patrick, and Steven A. Matthews. 1993. "Efficient and Nearly-Efficient Partnerships." Review of Economic Studies 60 (3): 599-611.

- Levhari, David, and Leonard J. Mirman. 1980. "The Great Fish War: An Example Using a Dynamic Cournot-Nash Solution.” Bell Journal of Economics 11 (1): 322-34.

-Ma, Ching-to, John Moore, and Stephen Turnbull. 1988. "Stopping agents from 'Cheating." Journal of Economic Theory 46 (2): 355-72.

-Mason, Robin, and Juuso Välimäki. 2015. "Getting It Done: Dynamic Incentives to Complete a Project." Journal of the European Economic Association 13 (1): 62-97.

-Marx, Leslie M., and Steven A. Matthews. 2000. "Dynamic Voluntary Contribution to a Public Project." Review of Economic Studies 67 (2): 327-58.

Moroni, Sofia. 2014. "Experimentation in Organizations." https://economics.nd.edu/assets/153487/ moroni_sofia_jmp.pdf.

Nikaido, Hukukane. 1968. Convex Structures and Economic Theory. Vol. 51, Mathematics in Science and Engineering. New York: Academic Press.

Olson, Mancur. 1965. The Logic of Collective Action: Public Goods and the Theory of Groups. Vol. 124, Harvard Economic Studies. Cambridge: Harvard University Press.

- Reinganum, Jennifer F., and Nancy L. Stokey. 1985. "Oligopoly Extraction of a Common Property Natural Resource: The Importance of the Period of Commitment in Dynamic Games." International Economic Review 26 (1): 161-73.

-Sannikov, Yuliy. 2008. "A Continuous-Time Version of the Principal-Agent Problem." Review of Economic Studies 75 (3): 957-84.

- Yildirim, Huseyin. 2006. "Getting the Ball Rolling: Voluntary Contributions to a Large-Scale Public Project." Journal of Public Economic Theory 8 (4): 503-28. 